University of Missouri, St. Louis

IRL@UMSL

Dissertations

UMSL Graduate Works

$12-9-2013$

\title{
Coping Styles among Individuals with Severe Mental Illness and Comorbid PTSD
}

Shannon Ashley McNeill

University of Missouri-St.Louis, samcneill@gmail.com

Follow this and additional works at: https://irl.umsl.edu/dissertation

Part of the Psychology Commons

\section{Recommended Citation}

McNeill, Shannon Ashley, "Coping Styles among Individuals with Severe Mental Illness and Comorbid PTSD" (2013). Dissertations. 277.

https://irl.umsl.edu/dissertation/277

This Dissertation is brought to you for free and open access by the UMSL Graduate Works at IRL @ UMSL. It has been accepted for inclusion in Dissertations by an authorized administrator of IRL @ UMSL. For more information, please contact marvinh@umsl.edu. 
Shannon A. McNeill

M.A., Clinical Psychology, University of Missouri - St. Louis, 2010

B.S., Psychology, Suffolk University, 2006

A Dissertation Submitted to The Graduate School at the University of Missouri - St. Louis in partial fulfillment of the requirements for the degree Doctor of Philosophy in Clinical Psychology

August 30, 2013

$\underline{\text { Advisory Committee }}$

Tara Galovski, Ph.D.

Chairperson

Ann Steffen, Ph.D.

Matthew Taylor, Ph.D.

Gary Morse, Ph.D.

Copyright, Shannon A. McNeill, 2013 
Coping Styles among Individuals with Severe Mental Illness and Comorbid PTSD

Shannon A. McNeill

University of Missouri - St. Louis 


\begin{abstract}
There is little known about the mechanisms by which persons with SMI cope with their stress, and virtually no research is available on the influence of comorbid PTSD (SMI-PTSD) on coping within the SMI population. The current study examined coping strategies utilized by individuals with SMI versus those with SMI-PTSD, while also investigating the role of PTSD symptom severity, overall psychological distress, and substance use on coping strategy usage. Participants included adults ( $N$ $=90)$ recruited through a metropolitan community mental health center, all describing current symptoms of an SMI, 48 of whom met criteria for SMI-PTSD. Results of this study revealed high rates of trauma exposure, with $83 \%$ of the total sample endorsing at least one traumatic event. Mean scores for posttraumatic symptom severity, psychological distress and substance abuse all fell within the ranges of clinical severity. Avoidance coping was used to a greater extent by the SMI-PTSD group as compared to the SMI group with no comorbid PTSD. PTSD symptom severity was a significant predictor of psychological distress experienced by trauma exposed participants. Additionally, for those exposed to trauma, avoidance coping partially mediated the relationship between their PTSD symptom severity and psychological distress. Substance use did not prove to be an influential variable in describing or predicting coping strategy use. These findings highlight the role of avoidance coping in the relationship between PTSD symptom severity and overall psychological distress within an SMI sample. Implications for treatment are considered.
\end{abstract}




\section{Acknowledgments}

Completion of my dissertation and resultant doctoral degree would not have been made possible without a select few who remain my steadfast cheerleaders. I am especially grateful to JoAnne McNeill and Eli Firicano, for their continued support, guidance and encouragement. 
By definition, individuals with severe mental illness (SMI) experience marked distress and significant limitations across multiple life domains (Mueser, Rosenberg, Hamblen, \& Decamps, 2004; National Alliance on Mental Illness, 2011; Peck \& Scheffler, 2002). Difficulties in social, occupational, leisure and elements of self-care are notable deficits that persons with SMI must navigate, but these are not the only stressors to be managed. Those with SMI also frequently experience substance abuse, financial constraints, as well as criminal victimizations (Classen, Palesh, \& Aggarwal, 2005; Drake et al., 2001; Drake, Mueser, Clark, \& Wallach, 2005; Fortier et al., 2009; Hultman, Wieselgren, \& Ohman, 1997; Mueser, Rosenberg, Goodman, \& Trumbetta, 2002; O'Hare, Sherrer, \& Shen, 2006; Subica, Claypoole, \& Wylie, 2012). In fact, victimizations and other traumatic events are experienced at such a rate that researchers have reported a heightened risk for trauma exposure and subsequent posttraumatic stress disorder (PTSD) among this population (Cascardi, Mueser, DeGiralomo, \& Murrin, 1996; Coverdale \& Turbott, 2000; Craine, Henson, Colliver, \& MacLean, 1988; Cusack, Grubaugh, Knapp, \& Frueh, 2006; Gearon, Kaltman, Brown, \& Bellack, 2003; Lommen \& Restifo, 2009; Mueser et al., 1998; Neria, Bromet, Sievers, Lavelle, \& Fochtmann, 2002; Subica et al., 2012). Furthermore, for many SMI individuals seeking services at community mental health centers (CMHCs), the presence of comorbid PTSD has been misdiagnosed and/or overlooked (Bonugli, Brackley, Williams, \& Lesser, 2010; Brady, Rierdan, Penk, Losardo, \& Meschede, 2003; Cusack et al., 2006; Grubaugh, Zinzow, Paul, Egede, \& Frueh, 2011; Lommen \& Restifo, 2009; Mueser et al., 1998). Previous investigations show that comorbid PTSD negatively influences a range of symptoms experienced by those 
with SMI, including depression severity, physical health complaints, and overall mental health (Mueser et al., 2002; Subica et al., 2012). Our increasing awareness of the prevalence of PTSD and the role this disorder may play in SMI symptom expression and exacerbation invites us to re-examine our assessment and treatment of these individuals (Grubaugh et al., 2011; Mueser et al., 2002).

The literature on psychosocial functioning and psychopathology suggests that those suffering from SMI are tasked with managing a range of psychosocial stressors, such as financial constraints, limited social networks, and criminal victimizations (Hiday, Swartz, Swanson, Borum, \& Wagner, 1999; Macdonald, Jackson, Hayes, Baglioni, \& Madden, 1998; Shelton, Taylor, Bonner, \& van den Bree, 2009; Teplin, McClelland, Abram, \& Weiner, 2005). Individuals with SMI also report increased stress as compared to healthy controls along a range of domains, including domestic issues, motivation, self-efficacy, and management of depression (Betensky et al., 2008). Additionally, psychophysiological evidence suggests that stress adversely impacts those with SMI, particularly schizophrenia, more so than healthy individuals (Betensky et al., 2008; Ganguli, Singh, Brar, Carter, \& Mintun, 2002; Kodesh et al., 2012; Kudoh, Ishihara, \& Matsuki, 1999). Given the particularly complicated nature of SMI across multiple domains of functioning and psychopathology, management of distress is a salient issue of investigation and treatment. It may prove to be an even higher priority for those with SMI and comorbid PTSD (SMI-PTSD) as surmounting evidence indicates that this population is at greater risk to engage in life-endangering behaviors following stressful events (O'Hare et al., 2006). They are also more likely to attempt suicide than those with SMI alone (Alvarez et al., 2012). We may be better 
positioned to assist and treat this burdened population that faces potentially lifethreatening circumstances by further investigating their stressors and how they cope with them.

To date, there is little known about the mechanisms by which persons with SMI cope with their stress, and virtually no research has been published on coping strategies within the comorbid condition of SMI-PTSD. The current study seeks to fill these gaps by investigating the coping strategies utilized by individuals with SMI versus those with SMI-PTSD. A number of variables that have the potential to influence coping, such as co-occurring substance use disorders (SUDs), criminal victimization, as well as demographic factors will be examined to provide a rich and detailed description of coping among those with SMI and SMI-PTSD. The possible relationships amongst these variables will also be examined to the same end.

Knowledge of the prevalence and role of coping could inform SMI treatment interventions and related areas of focus for clinicians, case-managers, and others working with SMI-PTSD persons. Additionally, an investigation into coping among SMI may illuminate some of the factors associated with the development of or even resiliency to comorbid PTSD — an area of research not yet developed, but greatly needed given the increased rates of trauma and PTSD.

\section{Severe Mental Illness}

\section{Defining SMI}

SMI is an umbrella term that encompasses a number of mental illnesses and is sometimes used in state parity laws to enable persons with certain mental illnesses to receive insurance and disability benefits (Peck \& Scheffler, 2002). SMI terminology 
is also commonly used by national agencies, local community mental health centers, and researching bodies to describe a particular subset of patients.

For the most part, there is agreement among national, state, and private agencies, as well as researchers, that the functional impairments related to an SMI involve serious limitations to a number of significant life domains including social, occupational, leisure, and self-care (see Mueser et al., 2004; Peck \& Scheffler, 2002). However, the named psychiatric disorders that are considered SMIs can differ among national and state service agencies/organizations and researching groups. For instance, Nebraska's Mental Health Board states that disorders qualifying as SMI are limited to schizophrenia, schizoaffective, bipolar, major depressive (MDD), and psychotic disorders (Nebraska Department of Health and Human Services, 2006). Meanwhile, the Ohio Department of Mental Health (2004) dictates that the following disorders are to be considered SMIs: "delusional disorders, dissociative disorders, eating disorders, mood disorders, organic mental disorders, personality disorders, psychotic disorders, schizophrenia, and/or somatoform disorder” (pp. 12-13).

National definitions of SMI, which are often used to investigate current prevalence and concordance rates, utilize more inclusive standards. For example, the National Institute of Mental Health (2010) classifies individuals as living with an SMI if they experience a work disability, substantial limitation, or role impairment due to a mental or substance disorder, non-affective psychosis, and/or bipolar I or II disorder within the past year, or if they have attempted suicide in the past year. Prevalence rates of SMI, as defined this way, are estimated to encompass approximately $6 \%$ of the American population (Kessler, Wai, Demler, \& Walters, 2005). This definition 
specifically names bipolar, substance, and psychotic disorders, but also indicates that a range of other mental illnesses are to be classified as SMI if they cause serious functional impairment and/or exacerbate distress enough to precipitate a suicide attempt. Another national organization, The National Alliance on Mental Illness (2011), provides examples of SMI, such as schizophrenia, bipolar disorder, MDD, obsessive compulsive disorder (OCD), panic disorder, PTSD, and borderline personality disorder. This varied catalog of accepted disorders, along with the insinuation that this may not be an exhaustive list, reflects a largely inclusive definition of SMI.

Although a variety of disorders, including anxiety disorders, are cited as SMIs per national definitions, some of these disorders may be less likely to accurately represent the majority of persons labeled as severely mentally ill and receiving services or disability benefits for their conditions. Within CMHCs, from which individuals with SMI are most likely to receive services (McAlpine \& Mechanic, 2000), it is more often the case that affective and psychotic disorders are described as the primary condition and qualifier for the SMI distinction. Some researchers have investigated the diagnostic enumerations from clinical inventories or chart reviews of persons deemed severely mentally ill and found elevated and sometimes exclusive rates of mood and psychotic disorders (Drukker, van Os, Bak, Campo, \& Delespaul, 2010; Grubaugh, Cusack, \& Zinzow, 2008). Furthermore, when researchers report their definition of SMI it is usually consistent with CMHC definitions.

In an abbreviated analysis of the studies utilized for reference in the current investigation, 20 out of 40 of the articles that specify their SMI inclusion criteria 
exclusively refer to schizophrenia/schizoaffective, bipolar, and MDD as the qualifiers for SMI. Comparatively, only 3 of the 40 articles extend their definitions to include anxiety disorders or other Axis I disorders. A more detailed configuration of the referenced articles and their corresponding SMI definitions are shown in Table 1. This is not to say that anxiety disorders are not often present among persons with SMI, in fact, many research findings assert otherwise (Buckley, Miller, Lehrer, \& Castle, 2009; Ciapparelli et al., 2007; Nebioglu \& Altindag, 2009). Nonetheless, anxiety disorders are not commonly recognized as primary conditions accounting for the SMI label. 


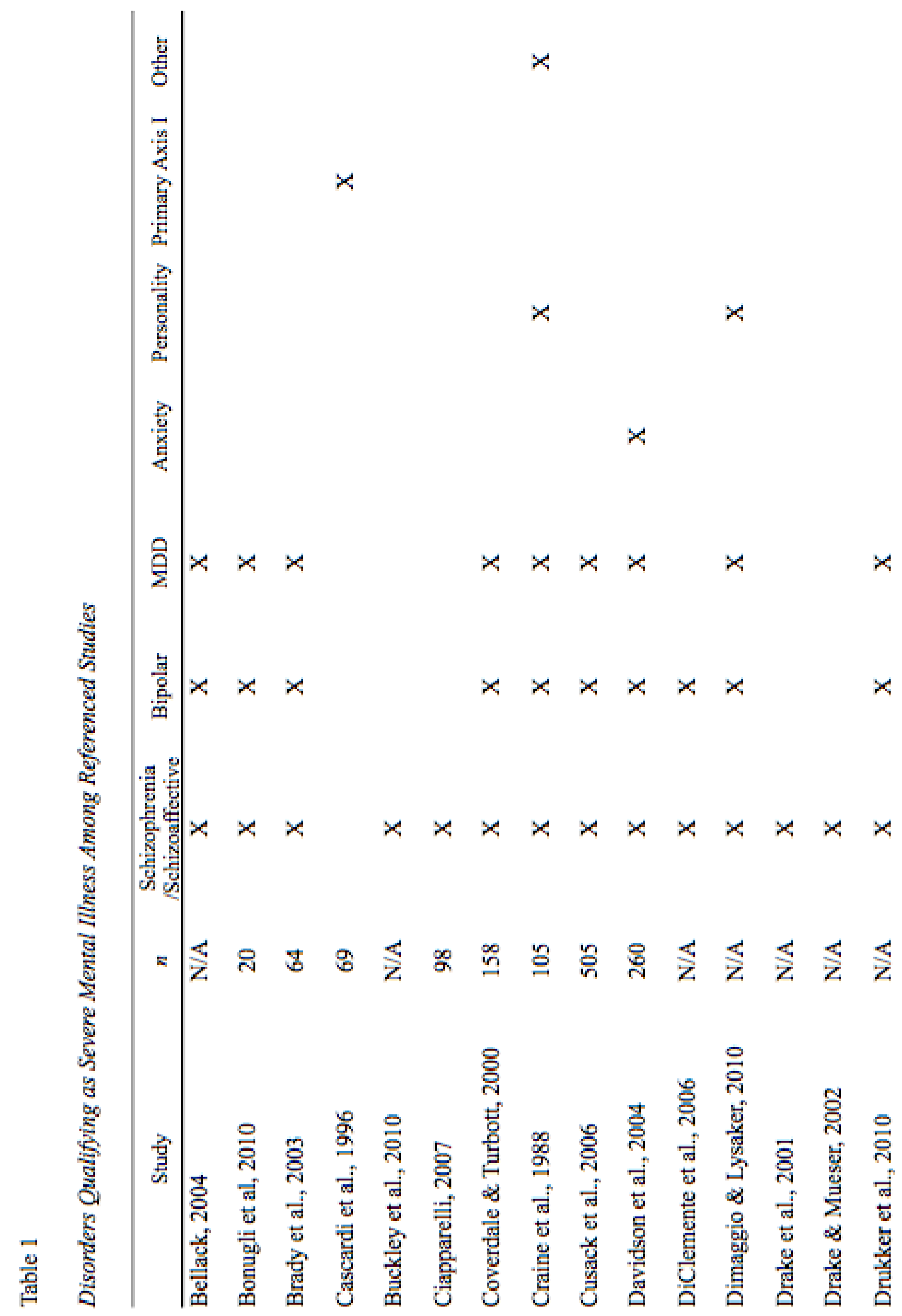




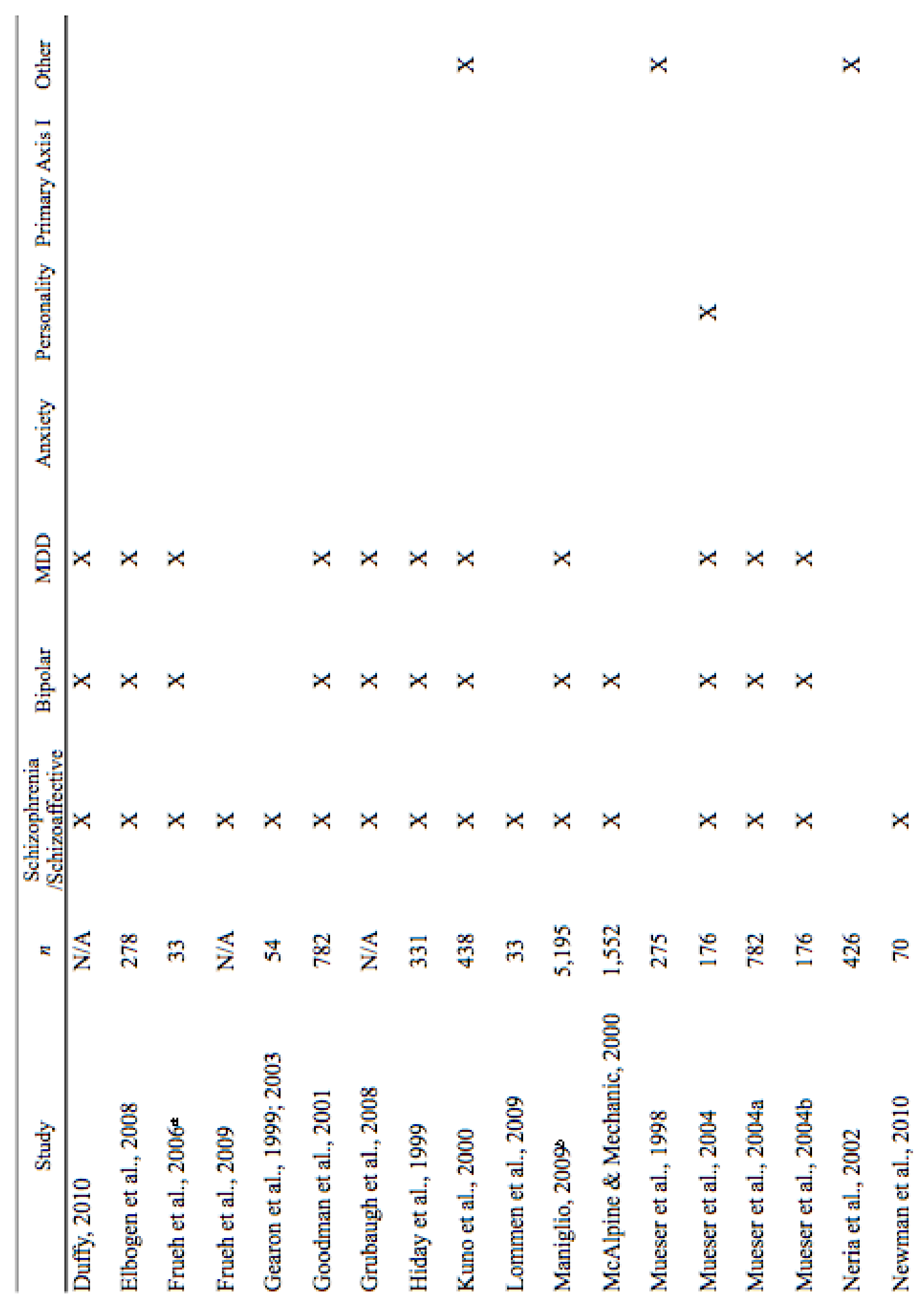




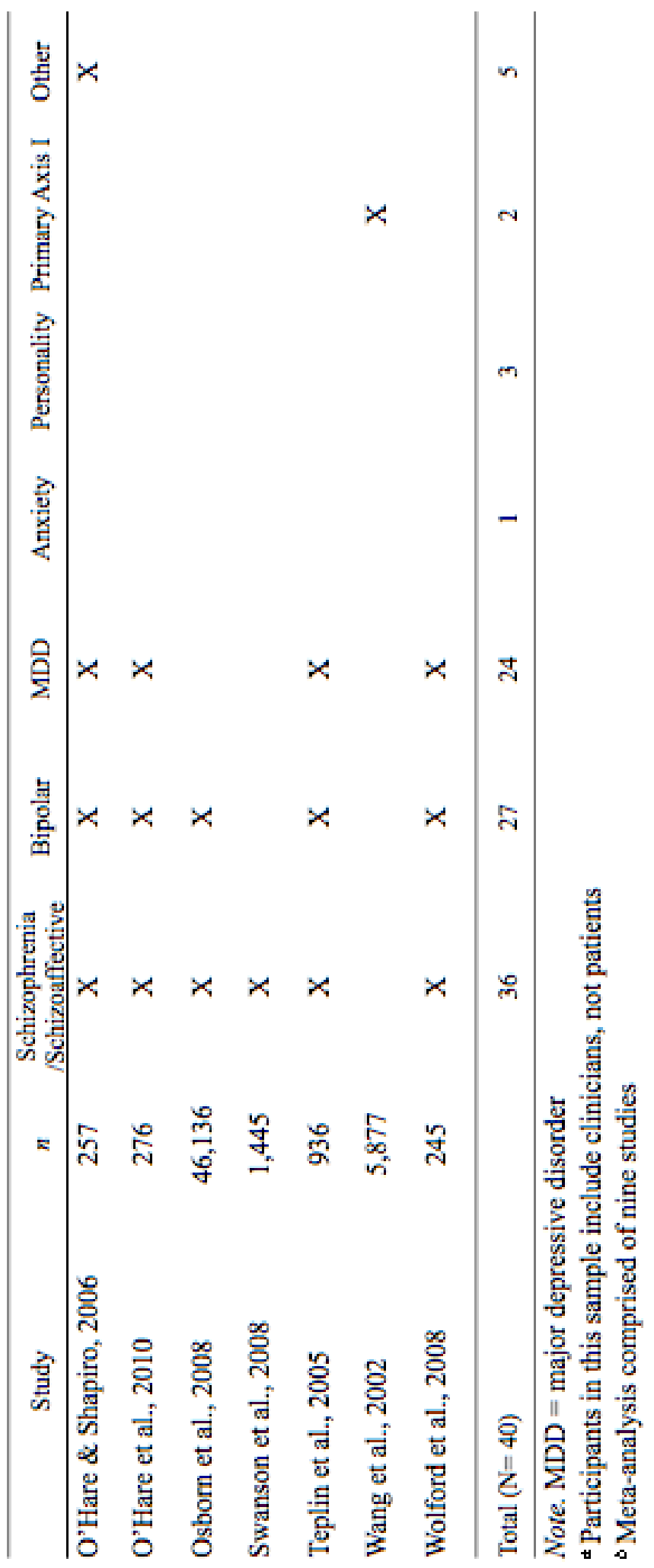


For the purposes of the current review and study, SMI will be defined as a psychiatric illness that significantly impairs one's functioning and may lead to qualification for social security or disability allowances. This is in accordance with the majority of SMI definitions represented by national, state, and research agencies. Additionally, in keeping with most CMHC definitions, and the majority of contemporary research investigating issues relevant to SMI, primary diagnoses including schizophrenia, schizoaffective disorder, schizotypal and schizoid personality, delusional disorder, MDD and bipolar I disorder with severe features will be considered SMI, while primary disorders of anxiety, such as PTSD will not be included in the definition.

\section{SMI and Coping}

Individuals suffering from SMI must manage a range of difficulties that significantly interfere in their ability to lead satisfying and productive lives. Their task of managing these stressors is two-fold: (1) coping with the direct symptoms related to their identified SMI, such as hallucinations or depression, and (2) coping with stressors that directly or indirectly arise from living with an SMI, such as homelessness and victimization (Burton, Chaneb, \& Meeks, 2007; Meyer, 2001; Phillips, Francey, Edwards, \& McMurray, 2009). Despite the increased potential for experiencing substantial stressors, the literature on coping within SMI is relatively under-developed (Yanos, Knight, \& Bremer, 2003). 


\section{Barriers to Researching Coping}

There are a number of challenges to investigating SMI and coping. First, the inclusion of individuals with SMI, or any human subjects, in research is usually contingent upon their mental capacity to understand the risks and benefits of participation. While no particular psychiatric disorder denotes mental incapacity to consent, individuals with SMI may be more vulnerable to misunderstanding or being coerced during the consenting process (Capron, 1999; Welie \& Berghmans, 2006). For non-therapeutic research involving no more than minimal risk to participants, such as the majority of investigations into coping styles, the issue of enrolling individuals with SMI into research is less concerning. Nonetheless, precautions need to be taken to confirm that those participating in research are competent to consent to their enrollment. National committees, such as the National Bioethics Advisory Commission (1998), that monitor and impart guidelines related to the protection of human subjects require objective assessment of individuals at risk for decreased mental capacity to consent, such as those with mental disorders. Objective assessments can include mental status examinations, preferably completed by a qualified individual not directly involved in the research (Capron, 1999). While individuals with SMI are not generally excluded from non-therapeutic research due to incapacity to consent (Welie \& Berghmans, 2006), their increased vulnerability for it represents a unique challenge to research, including that focused on coping.

A barrier to conducting research within the field of coping and stress management, in general, is the lack of measurement and methodological consistency (Phillips et al., 2009). A multitude of measures have been developed and utilized 
since coping with mental illness became a construct of significant scientific interest approximately 20 years ago (Farhall, Greenwood, \& Jackson, 2007; Meyer, 2001; Phillips et al., 2009; Yanos et al., 2003). These measures use different methodological approaches (open-ended questioning vs. categorical appraisal) to assess coping (Meyer, 2001). They also tend to use different terminology to define their coping strategy categories (Yanos et al., 2003). This can make cross-study comparisons and meta-analytic investigation particularly difficult — a significant barrier to producing meaningful and cohesive understandings of the extant literature.

Additionally, the majority of coping measures that are available have not been developed for the use with SMI populations, or have not yet been validated with this group (Meyer, 2001; Phillips et al., 2009; Yanos et al., 2003). In a general discussion of coping research, Coyne and Racioppo (2000) describe the dangers of employing a single measure of coping across multiple situations and varied populations. They explain that an "omnibus coping checklist" adds very little to our understandings of how individuals cope and whether changes in coping can lead to effective outcomes. Neglecting important individual and contextual characteristics such as gender, perceived goals, and psychological disorders produces findings that are too broad and add little descriptive or applied value to the overall field of coping research. Given the inherent severity and uniqueness of the SMI population, it follows that the specific stressors common to those with SMI and their related coping strategies should be measured independent of the general population (Coyne \& Racioppo, 2000; Yanos et al., 2003). Following Coyne and Racioppo's call to action, more SMIspecific coping measures have been developed, though few have accumulated the 
empirical support garnered by the previously mentioned "omnibus coping checklists." Nonetheless, the investigative interest into coping and SMI continues to grow (Yanos et al., 2003). Use of SMI-specific measures and replication of results using an SMI sample will contribute to the meaningful findings on the topic of coping, and subsequently translate to prevention and intervention research.

A number of coping strategies, such as avoidance coping, problem-focused coping, and neutral coping, have been investigated within SMI samples (Phillips et al., 2009). Two primary coping strategies have emerged as promising in the SMI literature-avoidance and problem-focused coping (Yanos, West, \& Smith, 2010).

\section{SMI and Avoidance Coping}

Avoidance coping has been described as a set of behavioral and cognitive responses to stress that are aimed at reducing the experienced anxiety by avoiding it (Lazarus \& Folkman, 1984; Pineles et al., 2011). Avoidance strategies can include using substances, social withdrawal, or intentionally focusing one's attentions away from the perceived source of the anxiety (Lazarus \& Folkman, 1984; Yanos et al., 2003). Avoidance coping is generally regarded as a maladaptive strategy that does not effectively mitigate distress or lead to long-term problem solving (Lazarus \& Folkman, 1984; Phillips et al., 2009). Researchers have found that persons with SMI who use avoidance coping strategies to deal with stressful events experience more negative mood, decreased productive use of time (increased time spent sleeping, watching TV, etc.), increased residual distress following natural disasters, increased schizophrenia symptoms, and heightened neuroticism trait levels (Horan et al., 2007; Lysaker, Wilt, Plascak-Hallberg, Brenner, \& Clements, 2003; Meyer, 2001; Yanos et 
al., 2010). While no singular coping strategy has been consistently associated with SMI, some researchers have demonstrated that those with SMI utilize avoidance coping more often than control comparison groups (Horan et al., 2007; Phillips et al., 2009).

\section{SMI and Problem-Focused Coping}

Problem-focused coping describes a person's use of specific strategies aimed at reducing their perceived distress by resolving or changing the objective variables of a situation. Individuals may use behavioral or cognitive problem-solving skills such as using social supports, evaluating one's options, making a decision, and acting upon the chosen decision (Sabina \& Tindale, 2008). The pro-active nature of this strategy indicates that it is generally considered a positive or adaptive coping strategy, particularly when the stressor is believed to be changeable (Holahan, Moos, \& Schaefer, 1996). Among persons with SMI, problem-focused coping strategies, such as the use of prescribed medication and social supports, have been associated with decreased negative mood and better social functioning (Yanos et al., 2003; Yanos et al., 2010). There are mixed findings on the prevalence of problem-focused coping among individuals with SMI. For example, Berry and colleagues (2006) found that outpatients with SMI were more likely to use problem-focused coping as compared to healthy controls. Conversely, Horan and colleagues (2007) demonstrated that an outpatient SMI sample reported using fewer active coping strategies (similar to problem-focused coping) than a group of non-psychiatric controls. These mixed findings could be the result of inconsistent terminology and methodology. Active coping is defined similarly to problem-focused coping, but the strategies have yet to 
be directly compared. As such, it remains unclear if these concepts are qualitatively similar. More research is needed to determine the prevalence and utility of problemfocused coping among those with SMI.

Most investigations into coping do not use a binary model to evaluate the strategies used. Doing so can decrease the specificity and subsequent meaningfulness of a study's results. In fact, those with SMI and within the general population tend to use a variety of coping strategies (see Phillips et al., 2009 for review). Furthermore, researchers have demonstrated that the perceived efficacy of one's ability to cope (i.e., how much one believes their coping is useful) is associated with increased coping strategy flexibility, or the ability to use a range of strategies to maneuver stressful situations (Penley, Tomaka, \& Wiebe, 2002; Phillips et al., 2009; Tarrier, 1987). As such, a number of other coping strategies have been evaluated among those with SMI, including emotion-focused, and neutral coping. However, these strategies have less empirical support as compared to avoidance and problem-focused coping (Phillips et al., 2009; Yanos et al., 2003).

\section{Trauma and PTSD Prevalence within SMI}

The available literature describing coping among those with SMI is helpful for conceptualizing SMI distress management, but falls short in describing the SMIPTSD experience. It is likely that the previously described studies of SMI and coping included many SMI-PTSD participants, but without direct investigation of the role of PTSD among SMI individuals we can draw guarded conclusions regarding coping and the SMI-PTSD population. 


\section{Trauma Exposure and SMI}

The reported incidence rates of trauma exposure among those suffering from an identified SMI vary greatly, with rates ranging from 54-98\% of persons experiencing at least one traumatic event in their lifetime (Cascardi et al., 1996; Coverdale \& Turbott, 2000; Cusack et al., 2006; Gearon et al., 2003; Lommen \& Restifo, 2009; Mueser et al., 1998; Neria et al., 2002; Subica et al., 2012). The lower bound of this range is reminiscent of reported rates of trauma exposure within the general population (39-56\%; Breslau, Davis, Andreski, \& Peterson, 1991; Kessler, Sonnega, Bromet, Hughes, \& et al., 1995), and suggests that those with SMI are at a similar risk of experiencing traumatic events as compared to most individuals. Table 2 describes the reported trauma exposure rates within the studies used to generate this range. After further inspection, it is found that the majority of these studies reflect trauma exposure rates higher than $85 \%$, with the median rate being $88 \%$. 
Table 2

Rates of Trauma Exposure and PTSD Prevalence among Severe Mental Illness Samples within Referenced Studies

\begin{tabular}{lcccc}
\hline \multicolumn{1}{c}{ Publication } & $\begin{array}{c}\text { SMI sample } \\
\text { size } \\
N\end{array}$ & $\begin{array}{c}\text { Lifetime trauma } \\
\text { exposure } \\
n(\%)\end{array}$ & $\begin{array}{c}\text { Current PTSD } \\
\text { confirmed } \\
n(\%)\end{array}$ & $\begin{array}{c}\text { PTSD reported in } \\
\text { clinic chart } \\
n(\%)\end{array}$ \\
\hline $\begin{array}{l}\text { Coverdale \& Turbott, } \\
2000\end{array}$ & 158 & $86(54 \%)$ & N/A & N/A \\
Gearon et al., 2003 & 54 & $50(96 \%)$ & $25(46 \%)$ & N/A \\
Mueser et al., 1998 & 275 & $270(98 \%)$ & $119(43 \%)$ & $3(2 \%)$ \\
Niera et al., 2002 & 426 & $292(69 \%)$ & $61(14 \%)$ & N/A \\
Bonugli et al., 2010 & 20 & $20(100 \%)$ & $20(100 \%)$ & 0 \\
Cascardi et al., 1996 & 69 & $42(61 \%)$ & $20(29 \%)$ & N/A \\
Craine et al., 1988 & 105 & $54(51 \%)$ & $36(34 \%)$ & 0 \\
Cusack et al., 2006 & 142 & $124(87 \%)$ & $42(30 \%)$ & $5(4 \%)$ \\
$\begin{array}{l}\text { Lommen \& Restifo, } \\
\text { 2009 }\end{array}$ & 33 & $32(97 \%)$ & $3-6(18 \%)$ & N/A \\
Brady et al., 2003 & 64 & N/A & $15(23 \%)$ & $2(3 \%)$ \\
Subica et al., 2012 & 175 & $149(89 \%)$ & $79(41 \%)$ & N/A \\
\hline Note: N/A $=$ not app & & & \\
\hline
\end{tabular}

Note: $\mathrm{N} / \mathrm{A}=$ not applicable

${ }^{a}$ this investigation does not specify it's population as SMI, rather it included those admitted to psychiatric hospital with psychosis and investigated lifetime PTSD diagnoses

${ }^{b}$ sample is exclusively female and selected based on endorsement of sexual abuse history \& no PTSD diagnosis recorded in clinic chart, as such these rates were not used to generate trauma and PTSD prevalence ranges

${ }^{c}$ sample is exclusively female; only childhood sexual abuse queried and reported, therefore these rates were omitted from trauma prevalence estimate ranges

While the lower bound of 54\% may be an accurate representation of trauma exposure rates within the SMI population, a number of factors could also explain its comparatively low percentage. First, Coverdale and Turbott's (2000) investigation, which reports the $54 \%$ prevalence, took place in New Zealand. While this is not the 
only international study included in the ranges, a number of cultural differences may be influencing the results. More investigation into issues such as the general population rates of trauma in New Zealand, as well as the cultural perception of trauma, and comfort with disclosure of traumatic events would provide more context for the findings. Additionally, the authors report that participants were asked about exposure to childhood physical and sexual abuse, while other traumas were not queried (Coverdale \& Turbott, 2000). Exclusive examination of particular trauma types likely contributes to the decreased rate of trauma exposure found in this investigation.

Given these considerations, and further examination of the spread of reported trauma exposure, it appears that individuals with SMI generally experience increased rates of traumatic events as compared to the general population. Of the types of traumatic experiences reported by individuals with SMI, physical and sexual abuse is often cited (Bonugli et al., 2010; Craine et al., 1988; Cusack et al., 2006; Neria et al., 2002). This is a notable point of interest, as these trauma types have been associated with increased PTSD rates among the general population (Scott, 2007). Coverdale and Turbott (2000) described increased reports of adult physical and sexual abuse among persons with SMI, compared to non-psychiatrically ill controls with similar age and ethnicity. These findings further illuminate the association between SMI and trauma exposure, and physical and sexual abuse more specifically. However, some sociodemographic features, such as marital and occupational status, as well as the amount of social welfare assistance received by participants, could not be reliably matched between the groups in this study. Such social and environmental differences 
may explain the significant reporting differences found between these groups. Nonetheless, elevated rates of trauma exposure within the SMI population remain an important consideration informing clinical assessment and treatment planning procedures for this population.

\section{PTSD and SMI}

Given the substantial rates of trauma exposure and increased reports of physical and sexual abuse (Bonugli et al., 2010; Craine et al., 1988; Cusack et al., 2006; Neria et al., 2002), it follows that PTSD prevalence among persons with SMI might also be high. Similar to trauma exposure rates, the rates of PTSD among individuals with SMI vary considerably, from 14-46\% (Cascardi et al., 1996; Craine et al., 1988; Cusack et al., 2006; Gearon et al., 2003; Lommen \& Restifo, 2009; Mueser et al., 1998; Neria et al., 2002; Subica et al., 2012). The wide ranges exhibited for both trauma exposure and PTSD among those with SMI highlights the importance of careful examination of the methodological differences between these investigations. This is particularly befitting in the absence of supporting epidemiological or meta-analytic data.

The studies from which the PTSD prevalence range was generated are also represented in Table 2. On the lower bound, a lifetime PTSD prevalence rate of 14\% was found among a sample of inpatients admitted for their first identified psychotic episode. The authors acknowledge the lower rates of PTSD in their sample as compared to others' (Mueser et al., 1998; Switzer et al., 1999), and consider the impact that socioeconomic characteristics and chronicity of illness may have played. The authors explain that "the majority of the current sample were not on public 
assistance and did not have a chronic disability" (Neria et al., 2002, p. 250). As such, this sample describes a particular subset of SMI individuals who might not be representative of the larger SMI or CMHC clientele. Even when considering the limitations of this particular investigation, the reported ranges of PTSD among those with SMI are significantly larger than those estimated within the general population (3.5-8\%; American Psychiatric Association, 2000; Kessler et al., 2005). Furthermore, researchers have found that among those with SMI endorsing lifetime experiences of trauma, $19-67 \%$ of them also report symptoms consistent with a current PTSD diagnosis (Bonugli et al., 2010; Craine et al., 1988). This is more than three-times as high as the estimates for PTSD related to the trauma-exposed general population (6.3\%; Kessler et al., 2005).

\section{Assessment and Diagnostic Inaccuracies}

Contemporary research indicates that trauma exposure and PTSD are not being evaluated or charted properly within CMHCs. Specific screening procedures for traumatic experiences have not traditionally been included in intake protocols at most CMHCs (Cusack, Frueh, \& Brady, 2004; Fallot \& Harris, 2001; Zanville \& Cattaneo, 2009). The intakes that are commonly utilized may focus heavily on assessing current distress and limitations related to a primary complaint by the client, or the most prevalent and distinguishable symptoms (Fallot \& Harris, 2001; Zanville \& Cattaneo, 2009).

Exclusion of trauma exposure screening questions may prohibit a complete and accurate assessment of clients' psychosocial history and current symptomatology. Important information may be lost, potentially resulting in the under-diagnosis of 
PTSD (or other trauma-related sequelae) and the impediment of appropriate treatment formulation. While the extant literature describes elevated rates of PTSD within SMI, most of these investigations also indicate that PTSD diagnoses are omitted from patient charts (Bonugli et al., 2010; Brady et al., 2003; Cusack et al., 2006; Lommen \& Restifo, 2009; Mueser et al., 1998). For example, Bonugli and colleagues (2010) found that among their sample of 20 women with SMI endorsing histories of sexual abuse, all met current criteria for PTSD, yet none had the diagnosis charted in their psychiatric inpatient unit records. The evidence of under-diagnosis (or lack of inclusion in documentation) of PTSD in CMHCs is sizable, and examples continue, with Cusack et al. (2006) finding that only 5 of 42 actual cases of PTSD were recorded, and Mueser et al. (1998) showing that an alarmingly low 3 out of 119 PTSD cases were documented in patient charts.

To date, PTSD diagnostic status and trauma variables have not been investigated in their relation to coping among persons with SMI. Direct inquiry into these variables may further our understanding of coping among SMI for at least two reasons. First, more specific descriptions of the samples being included in the studies is a crucial element to detecting significant and meaningful relationships between variables in the coping literature (Coyne \& Racioppo, 2000). Second, consideration of trauma exposure and PTSD symptomatology may help to distinguish some of the mixed findings from the previous coping and SMI studies. For example, studies of prevalence of avoidance and problem-focused coping strategies have demonstrated conflicting rates. Some note that avoidance is highest among SMI, where others describe problem-focused as the preeminent strategy (Berry et al., 2006; Horan et al., 
2007; Phillips et al., 2009; Yanos et al., 2010). Both of these strategies have also been described in the coping and PTSD/trauma literature (described below). Thus, consideration of the impact of PTSD on coping strategies among individuals with SMI could clarify the prevalence and role of particular coping strategies, which may ultimately better inform current assessment and treatment interventions for those with SMI-PTSD.

\section{PTSD and Coping}

Given the lack of research on SMI-PTSD and coping, one must draw upon previous findings from the separate literatures of SMI and PTSD to inform any theoretical assumptions and empirical hypotheses related to coping. The available research on SMI and coping has demonstrated that avoidance and problem-focused coping are among the most researched and relevant strategies used by those with SMI (Berry et al., 2006; Horan et al., 2007; Phillips et al., 2009). The literature on PTSD and coping also highlights avoidance and problem-focused coping strategies in describing how those with PTSD manage stressful events (Bokszczanin, 2003; Ford, 2011; Fortier et al., 2009; Green, Choi, \& Kane, 2010; Krause, Kaltman, Goodman, \& Dutton, 2008; Schuettler \& Boals, 2011).

\section{PTSD and Avoidance Coping}

Theoretically, avoidance coping can be easily associated with PTSD. In fact, avoidance is a designated cluster of symptoms contained in the diagnostic criteria for PTSD. Avoidance symptoms describe the active and persistent efforts made by those with PTSD to disengage and evade thoughts and emotions related to an experienced trauma (American Psychiatric Association, 2000; Foa, Zinbarg, \& Rothbaum, 1992). 
Individuals with PTSD may use both cognitive and behavioral strategies to avoid unwanted emotional and physical states (Amir et al., 1997; Walser \& Hayes, 2006). A cognitive strategy might include distraction or dissociation from thoughts about one's trauma. Alternatively, a behavioral technique could involve purposefully avoiding places or people that remind an individual of their trauma.

Avoidance is not only a notable symptom of PTSD, but is also considered a set of cognitive and behavioral coping strategies that maintain the disorder (Badour, Blonigen, Boden, Feldner, \& Bonn-Miller, 2012; Foa \& Rothbaum, 1998; Krause, Kaltman, Goodman, \& Dutton, 2008; Resick \& Schnicke, 1993; Walser \& Hayes, 2006). Understanding the importance of avoidance coping, some of the preeminent therapies for PTSD directly target these behaviors by encouraging experiential exposure and cognitive processing of the avoided stimuli (Pineles et al., 2011). Given the prevalent role of avoidance in PTSD, it is not surprising that much empirical evidence supports the association between PTSD and avoidance coping (Amir et al., 1997; Benotsch et al., 2000; Fortier et al., 2009; Galor \& Hentschel, 2012; Krause et al., 2008; Pineles et al., 2011; Schuettler \& Boals, 2011; Tiet et al., 2006). In a study of women exposed to intimate partner violence, Krause, Goodman, and Dutton (2008) found a strong relationship between PTSD symptom severity and participants' use of avoidance coping strategies. The researchers effectively demonstrated that increased avoidance coping was predictive of higher PTSD symptoms at the initial interview, but also at a 1-year follow-up visit. This speaks to the stability of avoidance behaviors among those with PTSD. Other researchers have noted that increased avoidance coping is not only related to PTSD symptom severity, but also heightens the risk for 
re-victimization (Fortier et al., 2009). Additionally, Pineles and colleagues (2011) found that for women recently exposed to a traumatic event, avoidance coping strategies were particularly interfering when coupled with increased sensitivity to trauma cues. The researchers noted that while heightened physical reactivity (i.e. increased heart rate) to trauma cues is expected among those who have recently survived a trauma, the use of avoidance coping interferes in one's recovery process and leads to increased PTSD symptom severity over time. Taken together, avoidance coping has strong theoretical and empirical ties to PTSD. It is not only related to the experience of living with PTSD, but also plays a role in the development and longterm maintenance of the disorder.

\section{PTSD and Problem-Focused Coping}

Investigations that describe the role of problem-focused coping on PTSD are less extensive and explicit than those related to avoidance coping. Recall that problem-focused coping is generally considered an adaptive strategy for mitigating distress. While findings from studies of SMI and coping seemed to support this perspective (Berry et al., 2006; Horan et al., 2007), results from investigations using trauma exposed and PTSD populations are less consistent. For example, a study of crime victims' coping as it related to their specific victimizations demonstrated that increased use of problem-focused coping was associated with more social support, but was also related to lower ratings of overall well-being (Green, Choi, \& Kane, 2010). While some studies indicate that problem-focused coping among those exposed to traumas generally leads to less overall psychological distress, decreased depression, and fewer dissociative experiences (Bokszczanin, 2003; Ford, 2011; 
Gutner, Rizvi, Monson, \& Resick, 2006), at least two other investigations have shown positive relationships between PTSD symptom severity and problem-focused coping (Glass, Flory, Hankin, Kloos, \& Turecki, 2009; Schnider, Elhai, \& Gray, 2007). Glass and colleagues (2009), who examined coping responses among individuals exposed to Hurricane Katrina, explained their unexpected findings. They reasoned that individuals facing unpredictable and uncontrollable trauma(s) may have initially used problem-focused strategies to remedy their distress and mitigate harm. The totality of the trauma may have been too extensive for the strategy to be effective, which ultimately led to increased psychological distress and greater PTSD symptom severity.

Of note, the two studies that found significant positive relationships between problem-focused coping and PTSD symptom severity both asked participants to reflect on a specific trauma and their methods of coping with that discrete event. In contrast, two of the three studies that found a significant negative relationship assessed coping in more general and trait-like terms. These methodological differences may indicate a disparity between the advantages of problem-focused coping during versus after a traumatic event and account for the mixed findings.

There are mixed findings on the prevalence and role of problem-focused coping for both SMI and PTSD populations. To date, no studies have demonstrated the influence of problem-focused coping on SMI-PTSD populations. Furthermore, no studies have sought to compare SMI and SMI-PTSD samples on this particular strategy. Direct comparisons of these populations could evidence meaningful results that clarify the prevalence and role of problem-focused coping. 


\section{Additional Challenges}

Individuals with SMI-PTSD often experience challenges beyond those directly related to their disorder symptomatology, such as co-occurring SUDs and criminal victimization (Goodman, Rosenberg, Mueser, \& Drake, 1997; Grubaugh et al., 2011; Mueser et al., 2002; Rosenberg et al., 2001). These variables have the potential to exacerbate symptom distress and could contribute to increasing one's risk for trauma exposure and subsequent PTSD development (see Mueser et al., 2002 for review). These elements may also impact the types and efficacy of coping strategies used by individuals with SMI.

\section{Substance Use}

SUDs are frequently reported as comorbid conditions for persons with SMI (Drake et al., 2001; Drake \& Mueser, 2002; Virgo, Bennett, Higgins, Bennett, \& Thomas, 2001). Additionally, research has indicated that adults suffering from PTSD are four to five times more likely to experience a comorbid SUD as compared to those without PTSD (Chilcoat \& Menard, 2003). Researchers describe an additive risk of experiencing PTSD for individuals with comorbid SMI and SUD. For example, Gearon and colleagues (2003) found that PTSD rates were higher in their sample of women with schizophrenia and comorbid SUD as compared to (1) the general population, (2) individuals with schizophrenia without SUD, as well as in (3) samples of women with SUD but no concurrent schizophrenia. Additionally, a reported history of childhood abuse among those with an SMI is described as a potential risk factor for the subsequent development of a SUD, though this pathway 
for SUD development remains somewhat unclear for the SMI population (Goodman et al., 1997; Meade, Kershaw, Hansen, \& Sikkema, 2009).

For individuals with a dual diagnosis of SMI and SUD, the impairments can be substantial. These individuals report poorer psychosocial functioning, increased rehospitalization, relapse, homelessness, and repeated incarceration rates (Baillargeon et al., 2010; see Drake et al., 2005 for review; Swofford, Kasckow, Scheller-Gilkey, \& Inderbitzin, 1996). The effects of substance intoxication can greatly interfere in one's ability to make decisions related to safety and can increase the risk for criminal victimization (Meade et al., 2009). Disabled judgment due to substance use can be particularly harmful for those with SMI, who may be contending with neurocognitive limitations that can affect their decision-making at baseline (Gearon \& Bellack, 1999; Maniglio, 2009).

Substance use, with the intention of reducing current anxiety or stress, is identified as an avoidance coping strategy (Lazarus \& Folkman, 1984; Yanos et al., 2003). Drinking as a demonstration of avoidance coping has been shown to mediate the relationship between PTSD symptom severity and alcohol use disorders (O'Hare \& Sherrer, 2011; Ullman, Filipas, Townsend, \& Starzynski, 2005; Yeater, Austin, Green, \& Smith, 2010). This indicates that individuals with problematic patterns of substance use, alcohol in particular, are likely to have increased PTSD symptom severity when their intended use of the alcohol is to aid in avoidance coping. Researchers have also found that increased PTSD symptom severity among those with SMI is associated with greater use of substances to cope with distressing events and/or beliefs (O'Hare, Shen, \& Sherrer, 2010). Though not directly investigated in 
O'Hare and colleagues' (2010) study, this indicates that the experience of PTSD may be more likely to increase one's use of avoidance coping via substance use than the experience of SMI symptomatology alone.

\section{Criminal Victimization}

The lifetime incidence of violent victimization for those with SMI is 2-11 times greater than that experienced by the general population (Hiday et al., 1999; Maniglio, 2009; Teplin et al., 2005). Assessments of the recency of criminal victimizations of people with SMI indicate that violent victimizations are an ongoing threat, as $33 \%$ of women and $37 \%$ of men with SMI report physical or sexual assaults happening within the past year (Goodman et al., 2001). In fact, the experience of having an SMI is described as a risk factor for re-victimization, particularly sexual assault re-victimization (Classen et al., 2005). Elements of one's victimization or trauma experiences have not been directly investigated or assessed within studies of SMI. Within the PTSD and coping literature, child sexual assault has been linked to increased use of avoidance coping and subsequent elevated symptom severity (Fortier et al., 2009), but other trauma variables including the number of different types of victimizations/traumas (child sexual assault, adult physical assault, witnessing death of another, etc.) have not been thoroughly investigated.

\section{Current Study}

Findings from the extant literatures on coping among those with SMI and PTSD provide insight into how these groups manage the many stressors they experience; however, there are limitations to these investigations. Specifically, the role of PTSD among the SMI samples is not addressed. Given the increased 
likelihood for those with SMI to have comorbid PTSD, it can be presumed that many of these participants carried an SMI-PTSD diagnosis. It is unclear how the addition of PTSD influences how an individual with SMI negotiates challenges and stressors. A study that accounts for PTSD diagnostic status among those with SMI could clarify this question.

Given that PTSD is closely affiliated with avoidance coping, it is likely that the presence or absence of PTSD greatly impacts how an SMI sample copes with their psychiatric symptoms and stressful life events. The current study seeks to test this supposition by directly comparing those with SMI, and no additional PTSD diagnosis, against those with SMI-PTSD on measures of coping and psychological distress. The role of additional challenges faced by individuals with SMI and SMIPTSD, such as substance use and criminal victimization, will be investigated by testing the impact of these variables on coping strategies between the groups. These larger objectives will be accomplished by enacting the following specific aims.

\section{Specific Aims and Hypotheses}

Aim 1. The first aim of the current investigation was to assess group differences in avoidance and problem-focused coping styles. This was done by comparing the results of self-report measures of coping between the two groups (SMI vs. SMI-PTSD). Coping was examined in terms of one's management of symptoms related to an identified SMI, as well as coping with an individualized recent stressor. This multiple assessment of coping provides in depth information about potential differences in coping strategies among those with SMI and SMI-PTSD. The following hypotheses describe the expected findings from these comparisons: 
Hypothesis 1a. It was hypothesized that differences between SMI and SMIPTSD samples would emerge on measures of avoidance coping. Specifically, participants with an SMI-PTSD diagnosis would demonstrate increased avoidance coping as compared to those with SMI alone on measures of coping with SMI symptoms (CSC: avoidance subscale) and coping with recent stressors (Brief-COPE: avoidance subscale).

Hypothesis $\mathbf{1 b}$. It was hypothesized that differences between SMI and SMIPTSD samples would emerge on measures of problem-focused coping. Specifically, participants with an SMI alone diagnosis would report increased use of problem-focused coping as compared to the SMI-PTSD group on measures of coping with SMI symptoms (CSC: problem-focused subscale) and coping with recent stressors (Brief-COPE: problem-focused subscale).

Aim 2. The second aim of the present study was to investigate differences in psychological distress as a function of PTSD diagnosis. Following the demonstration that those with SMI-PTSD report more psychological distress, the intention was to understand the role of coping in the relationship between PTSD symptom severity and psychological distress.

Hypothesis 2a. It was hypothesized that on measures of overall psychological distress, individuals with SMI would report lower psychological distress as compared to their SMI-PTSD counterparts.

Hypothesis 2 b: It was hypothesized that for individuals who have experienced a trauma, regardless of whether they met criteria for PTSD, PTSD 
symptom severity would predict overall psychological distress indirectly through avoidance coping.

Aim 3. The primary objective of the third aim was to investigate differences in substance abuse as a function of PTSD diagnostic status, and better understand the role of substance abuse in the relationship between PTSD symptom severity and avoidance coping separately for the groups. A secondary aim involved an examination of the relationship between criminal victimization and coping strategy use.

Hypothesis 3a: It was hypothesized that those with SMI-PTSD would show greater problematic use of substances compared to their SMI counterparts, such that a total substance abuse score would predict group membership.

Hypothesis 3b: It was hypothesized that PTSD symptom severity would predict avoidance coping indirectly through substance abuse for the SMIPTSD group only.

Hypothesis 3c: Exploratory analysis of the relationship between victimization variables and coping strategies were conducted without specific directional hypotheses. These exploratory analyses were expected to aid in the understanding of how trauma exposure potentially affects coping. The relative absence of literature on the relationships between trauma variables and coping allowed for exploratory investigations without direct hypotheses. 


\section{Method}

\section{Participants}

Ninety male and female adults participated in the current study. All participants were recruited through Places for People, a community mental health center based in St. Louis, Missouri that serves individuals at-risk or currently experiencing homelessness, mental illness and/or SUDs.

Eligible participants for the SMI group $(n=42)$ included individuals who were deemed competent to consent and had a current SMI (includes schizophrenia, schizoaffective, schizotypal or schizoid personality disorder, psychotic disorder not otherwise specified, delusional disorder, bipolar I, and/or MDD that meet "severe" status) but no PTSD. Competency to consent was evaluated with a brief mental state examination. Current SMI status was confirmed with a structured clinical diagnostic interview. Exposure to traumatic events was not exclusionary criteria for this group. The prevalence rate for trauma exposure among individuals with SMI is high, with estimates ranging from 54-98\% (Cascardi et al., 1996; Coverdale \& Turbott, 2000; Cusack et al., 2006; Gearon et al., 2003; Lommen \& Restifo, 2009; Mueser et al., 1998; Neria et al., 2002). This indicates that recruitment of a non-trauma exposed SMI group would have been (1) difficult to assemble, and (2) unlikely to represent the larger SMI population. The inclusion of trauma exposure in the SMI group likely did not obscure differences between the two groups. Epidemiological data shows that a significant proportion of the general population experience traumatic events, but do not go on to develop PTSD (Breslau et al., 1991; Kessler et al., 1995). The theoretical basis of PTSD asserts that those with PTSD are phenomenologically disparate from 
most people who experience a traumatic event, and an extensive amount of empirical data confirms this (American Psychiatric Association, 2000; Breslau et al., 1991; Kessler et al., 1995; Kessler et al., 2005). Furthermore, Vogel and colleagues (2006) demonstrated that psychological distress reported by those with SMI is significantly more influenced by PTSD symptoms than the experience of trauma itself. As such, the inclusion of those with trauma exposure and no PTSD appropriately represents the larger SMI population, while allowing for potential group differences to emerge. Within the collected SMI sample, 27 (64\%) participants reported experiencing a 'Criteria A' traumatic event at some point during their lifetime, while the remaining $15(36 \%)$ described no extreme traumatic stressors.

The SMI-PTSD group $(n=48)$ included participants who were deemed competent to consent and met criteria for a current SMI, as well as PTSD. PTSD diagnostic status was evaluated using a structured diagnostic interview. A self-report measure of PTSD symptom severity was also administered in order to obtain both categorical and continuous descriptions of the disorder.

\section{Procedure}

Participants were included in the study after completing a mental health assessment with the Places for People Mental Health Specialist and it was confirmed that they met current diagnostic criteria for SMI or SMI-PTSD. Eighty-five of the ninety participants recruited completed diagnostic assessments with the principal investigator. The remaining five participants were assessed by a research assistant with significant clinical experience. The research assistant received training by the principal investigator on the diagnostic instruments, and participated in clinical 
supervision of his diagnostic work by a licensed clinical psychologist. Statistical examination of differences based on assessor indicated that the participants evaluated by the research assistant were less likely to be diagnosed with PTSD than those evaluated by the principal investigator, $\chi^{2}(1)=6.05, p<.05, \Pi=.26$. This is a moderate association based on the size of the sample (Rea \& Parker, 1992). While this could be indicative of a discrepancy in diagnostic assessment between the assessors, it may also reflect the unpredictable nature of recruitment between SMI and SMI-PTSD groups. During the course of the principal investigator's data collection there were also chronological spans of recruitment of up to five participants in a row presenting with SMI alone diagnoses. The data collected by the research assistant were determined to reflect a group of participants similar to those previously collected by the principal investigator and were included in the total sample.

Recruitment and informed consenting was facilitated after the diagnostic interview portion of the assessment was completed. This procedure was done to (1) assuage potential frustration from ineligible clients completing the assessment with hopes of study participation, and (2) to reduce the potential for false reporting during the diagnostic assessment - either due to clients' desire to be seen as eligible or ineligible participants.

Informed consent. Potential participants were provided with a consent form describing the objective of the study, tasks involved, and potential risks and benefits associated with participation. A 3-item verbal quiz was administered to each participant after they read through the consent. The quiz assessed the participants' understanding of the material covered in the consent and their role as participants. 
Participants who responded incorrectly were provided with the correct responses and the quiz was re-administered. Though protocol instructed that participants who fail to respond correctly to the items a second time would be excluded from participation due to possible incapacity to consent, no participants failed twice.

Completion of study materials. Eligible and interested participants completed six self-report measures in addition to the interviews and measures contained in the standard Places for People mental health assessment. Completion of the additional measures added approximately 45 minutes to their typical assessment. Participants completed the full assessment and additional measures in a total of 2-2 $1 / 2$ hours. As compensation for their completion of the study portion of the assessment, participants were provided with $\$ 10$ cash. Data from the full assessment were used in the study as per participant's consent.

Difficulty reading and writing was predicted to be a concern for a portion of potential participants. Illiteracy did not preclude clients from participating in the current investigation. All participants were given the option of having the assessor read the self-report items aloud. Additionally, participants who demonstrate difficulties in reading and writing via a brief mental state examination were read the consenting materials and self-report items.

Protection against risk. As previously described, researchers enrolling individuals with SMI in their investigations should take additional efforts to confirm that potential participants evidence appropriate mental capacity to consent (Capron, 1999; National Bioethics Advisory Commission, 1998). Consistent with this ethical consideration, all potential participants were administered the Mini Mental State 
Examination (MMSE; Folstein, Folstein, \& McHugh, 1975) to confirm orientation to person, place, and thing, as well as fundamental reading and writing capabilities. This measure was administered before initiating the diagnostic interview and therefore preceded the consenting process. Clients who evidenced appropriate cognitive awareness to consent to research participation were offered the opportunity to participate in the current study.

During the consenting process participants were informed that completion of the interview and self-report measures should not have any harmful effects, however, answering questions about one's personal and traumatic experiences may be uncomfortable. It was explained that participants could stop answering the questions and withdraw from the study at any point. They were further informed that for any participant who became increasingly distressed at the time of interview, or while completing the questionnaires, opportunity to speak with a clinician or caseworker at Places for People would be made available. All participants were supplied with a phone number to an emergency hotline (Behavioral Health Response). During the course of recruitment no participants indicated a desire to withdraw from study activities during active participation. One participant contacted the principal investigator five months after completing the study requesting that their associated data be withdrawn. The request was immediately granted and both the participant and University of Missouri - St. Louis Institutional Review Board were notified of the action.

All information collected was kept confidential. Participants were assigned a subject identification number that was associated with their completed forms and 
interviews. The master file that linked subjects' identification numbers to their names and contact information was maintained on a Places for People computer server that was only accessible by registered staff. Furthermore, the file was encrypted and password protected. Only the principal investigator and trained research staff had access to the electronic document.

While information and data gathered during the study was confidentially maintained in the previously described ways, a portion of this data was used to complete the mental health assessment requested by Places for People. A written report that included diagnostic and psychosocial assessment information was generated to provide a thorough and clinically relevant description of the client and their reported difficulties. This document was placed in the client's Places for People file, which is accessible to case-managers, psychiatrists, and other Places for People staff who need the information to appropriately provide services to the client. This was in accordance with the approved file maintenance protocol for Places for People. The data generated by the measures that were added to the standard Places for People mental health assessment were stored separately. These data have been maintained on the University of Missouri- St. Louis campus at the Center for Trauma Recovery. The Center for Trauma Recovery is a locked facility that has secure hard and electronic data storing capabilities. Furthermore, the Center for Trauma Recovery is monitored by and compliant with the strict guidelines enforced by the University of Missouri St. Louis data safety monitoring board. 


\section{Power Analysis}

Apriori power analyses for each of the tests of hypotheses were conducted prior to data collection procedures to determine minimum sample size for the current investigation. Results demonstrated that to achieve appropriate power $(80 \%)$ to detect a medium effect $\left(f^{2}=.25\right)$ at the 0.05 level, hypotheses $1 \mathrm{a}, 1 \mathrm{~b}$, and $2 \mathrm{a}$ would require a total of 66 participants. Hypotheses $2 \mathrm{~b}$ and $3 \mathrm{~b}$ required at least 68 participants in total. Hypothesis 3a, a binary logistic regression with 1 predictor, represented the most complex analysis and required a total sample size of 113 to demonstrate a moderate effect. The anticipated sample for the current study was initially projected to include 150 participants. However, due to time constraints on data collection procedures a smaller sample of 90 participants was collected.

\section{Measures}

\section{Mental State.}

Mini Mental State Exam. The Mini Mental State Exam (MMSE; Folstein et al., 1975) is a brief screening tool for cognitive impairment. It contains tasks that evaluate 8 domains of cognitive functioning including orientation to time, orientation to place, registration, attention and calculation, recall, language, repetition, and complex commands. Out of a possible 30 points, scores $\leq 25$ are considered nonnormative and mental capacity may be compromised. This cut-off was used in the present study to determine capacity to consent. The MMSE has been found to appropriately predict capacity to consent to research participation, power of attorney, and placement in residential care (Whelan, Oleszek, Macdonald, \& Gaughran, 2009). 
Demographics. Basic demographic information including age, sex, race/ethnicity, insurance type, housing status, and length of time homeless (if applicable) was gathered using the standard Demographics Questionnaire (DQ) administered upon intake at Places for People. This 6-item questionnaire was supplemented with seven additional questions that are not reported on the Places for People DQ: education, relationship status, medication use (past and present), psychological treatment (past and present), number of identifiable social supports (noting family and non-family members), legal status, and personal report of legal history.

\section{Diagnostic.}

Structured Clinical Interview for DSM-IV Axis I Disorders. The Structured Clinical Interview for DSM-IV Axis I Disorders (SCID-I; First, Spitzer, Gibbon, \& Williams, 1996) is a semi-structured clinician-administered measure used to assess the presence of Axis I disorders. The following modules were administered to generate clinical profiles of the participants: mood, anxiety, psychotic, and substance disorders. For the purposes of the current study, and in accordance with the mental health assessment protocol at Places for People, current and past disorders were assessed. While the SCID-I is a straightforward, clinician-friendly tool, it requires the administrator to use clinical judgment to arrive at accurate diagnoses. The principal investigator conducted 85 of the interviews in the current study and the research assistant conducted 5 interviews. The principal investigator has over 5 years of clinical assessment experience and has administered over 150 SCID-I assessments. 
The research assistant has over 3 years of clinical assessment experience and administered three SCID-I assessments during his training on this study.

\section{Structured Clinical Interview for DSM-IV Axis II Personality Disorders.}

The Structured Clinical Interview for DSM-IV Axis II Personality Disorders (SCID-

II; First, Gibbon, Spitzer, Williams, \& Benjamin, 1997) is a semi-structured clinicianadministered tool used to diagnose the 10 DSM-IV Axis II personality disorders. Reliability tests of the SCID-II for DSM-IV indicate that the measure has moderate to high kappa levels between raters $(k=0.65-0.97)$ (Lobbestael, Leurgans, \& Arntz, 2011).

Scoring of the SCID-I and SCID-II generated indicators of the presence or absence of all diagnoses assessed for each participant. Additionally, participants were grouped into categories based on their primary disorder presentation. All participants fell into either the depressive-spectrum (i.e., MDD, bipolar I disorder, depressive disorder not otherwise specified, dysthymic disorder) or psychotic-spectrum disorder (i.e., schizophrenia, schizoaffective, delusional disorder, psychotic disorder not otherwise specified, schizoid personality disorder, schizotypal personality disorder) groups with no overlap between them.

\section{Coping.}

Coping with Symptoms Checklist. The Coping with Symptoms Checklist (CSC; Yanos et al., 2003) is a clinician-administered questionnaire specifically designed to assess coping strategies employed by individuals with SMI within the past 12 months. The measure examines coping across five areas of symptom distress commonly experienced by those with SMI: anxiety, depression, paranoia, 
hallucinations, and mania. Participants who do not endorse the presence of symptoms contained in one of the five areas are not asked subsequent questions relevant to that area. For example, if a participant denies having paranoid beliefs in the past 12 months, they will not be asked about their coping strategies related to paranoia. Coping strategies are organized into 3 categories: avoidance, problem-focused, and neutral. Mean scores for each coping strategy are generated within each domain of symptom distress. Overall scores for the 3 coping strategies are also calculated. Previously published internal consistency rating for each of the 3 coping strategies were all within acceptable to high ranges $(\alpha=.67-.88)$, with the exception of avoidance coping for the symptom combination of anxiety, depression, mania, hallucinations, and delusions ( $\alpha=.52$ ) (Yanos et al., 2003). Previously published tests of convergent and discriminant correlations reveal that the measure is a valid indicator of coping (Yanos et. al., 2003). For the current study, only the avoidance and problem-focused coping subscales were used in analyses. These subscales demonstrated acceptable internal consistency ratings ( $\alpha=.84$ and .91 , respectively).

Brief-COPE. The Brief-COPE (Carver, 1997) is a 28-item self-report measure that was adapted from an original full-length coping measure known as the COPE (Carver, Scheier, \& Weintraub, 1989). The items are formatted in a 4-point likert scale rating system that describes the frequency of coping strategy use. Three aggregate subscales are calculated from the original 14 subscales that the measure is capable of generating (problem-focused coping, active-emotional coping, and avoidant-emotional coping). These subscales were previously generated and used by Schnider and colleagues (2007), based on their theoretical applications from the full- 
length COPE and works of pioneer coping researchers (Carver et al., 1989; Lazarus \& Folkman, 1984). When applied to their sample, the three subscales evidenced strong internal consistency ratings (problem-focused $=.80$, active-emotional $=.81$, avoidantemotional $=.88)($ Schnider et al., 2007). The Brief-COPE has been validated with multiple populations including trauma-exposed, students, caretakers of those with dementia, and healthy controls (Carver, 1997; Cooper, Katona, \& Livingston, 2008; Yusoff, 2010). It has also been used with SMI samples to measure general, situation specific, and symptom specific coping styles (Meyer, 2001; Pruessner, Iyer, Faridi, Joober, \& Malla, 2011; So \& Wong, 2008). In his instructions for the use and utility of the Brief-COPE, Carver (1997) encourages researchers to modify the semantics of the measure's instructions and likert rating descriptions to appropriately reflect the sample of interest. As such, the Brief-COPE has been applied to many different populations and instruction sets vary from asking participants to focus on a specific event to stressful events more generally (Krageloh, 2011). For the purposes of the current study, participants were asked to describe and focus on a particularly stressful event they have experienced in the past month and answer the items based on how they coped with it. Orienting participants to a specific personal event will help them to accurately report on the coping methods they used, whereas vague instructions may be more likely to elicit responses influenced by self-concept or how one perceives themselves to cope with stress. For the current study, only the avoidance and problem-focused coping subscales were used in analyses. These subscales demonstrated acceptable internal consistency ratings ( $\alpha=.73$ and .82 , respectively). 


\section{Symptom distress.}

PTSD Checklist - Specific. The PTSD Checklist - Specific (PCL-S; Weathers, Litz, Herman, Huska, \& Keane, 1993) is a 17-item self-report measure that assesses the presence and symptom severity of PTSD based on the exposure to a specific traumatic event. Subscales for the PCL-S include each of the three diagnostic clusters reported in the Diagnostic and Statistical Manual of Mental Disorders-Fourth Edition, Text Revisions (DSM-IV-TR; American Psychiatric Association, 2000): reexperiencing, avoidance, and hyperarousal. Internal consistency ratings from previous investigations are strong among the subscales $(\alpha=.94, .85, .85$, respectively) (Ruggiero, Del Ben, Scotti, \& Rabalais, 2003). Symptom severity is assessed using a 5-point likert rating $(1=$ not at all to $5=$ extremely). Summation of the scored items generates a total severity score, which can range from $17-85$. Each of the 17 items on the PCL-S corresponds to a specific DSM-IV diagnostic symptom of PTSD. A symptom meets diagnostic criteria and counts towards the presence of PTSD if the severity is rated as 3 (moderately) or above. A total symptom severity cut-off score of 44, along with confirmation of required minimum symptom presence for PTSD diagnostic criteria is recommended to achieve optimal sensitivity and specificity of the PTSD diagnosis (Blanchard, Jones-Alexander, Buckley, \& Forneris, 1996; Ruggiero et al., 2003). When these scoring guidelines are used, the PCL-S has high diagnostic efficiency and acuity (.95) (Blanchard et al., 1996; Ruggiero et al., 2003). The PCL-S has been used and validated with SMI samples (Cusack et al., 2006; Mueser et al., 1998; Mueser et al., 2001). Mueser and colleagues (2001) examined the psychometric properties of the measure with their sample of 30 SMI outpatients who 
were receiving services at a local CMHC. The researchers reported finding strong internal consistency for the total severity score $(\alpha=.94)$, moderate reliability rates through test-retest comparisons ( $r=.60$ and $80 \%$ agreement $)$, as well as moderate convergent validity with other measures of PTSD ( $r=.67$ and $83 \%$ agreement). For the current study, the PCL-S total severity score was used in analyses. The measure demonstrated a strong internal consistency rating $(\alpha=.95)$.

Brief Symptom Inventory. The Brief Symptom Inventory (BSI; Derogatis, 1975 ) is a widely used self-report measure of psychological distress. Fifty-three items are rated on a 5 -point likert scale $(0=$ not at all to $4=$ extremely $)$. Nine primary subscales of distress are generated and provide information on severity of distress related to somatization, obsessive-compulsive, interpersonal sensitivity, depression, anxiety, hostility, phobic anxiety, paranoid ideation, and psychoticism. In addition to these subscales, three total indices of symptom presence and distress are generated. The global severity index (GSI) describes a respondent's overall distress, the positive symptom total (PST) score reports on the number of symptoms experienced, and the positive symptom distress index (PSDI) describes the level of distress experienced by the symptoms endorsed. The BSI was developed as a shortened version of the Symptom Checklist-90-Revised (Derogatis, 1983), a well validated and widely used measure of psychological distress. Previously published psychometric evaluations of the BSI indicate that its subscales have good internal consistency (alphas ranging from .71 to .85$)$ and the global severity index has good test-retest value (.90). A global severity index T-score of 63 indicates clinical severity range (Derogatis, 1975). The validity of the BSI is excellent, as it demonstrates good convergent and divergent 
properties (Derogatis \& Melisaratos, 1983). Furthermore, the BSI has been shown to be a good measure of psychological distress among SMI populations and across ethnicities (Hoe \& Brekke, 2008). The current investigation used the depression, psychoticism, and global severity indices to report on symptom specific and overall psychological distress of the collected sample. These subscales demonstrated acceptable ranges of internal consistency ratings $(\alpha=.80-.98)$.

Modified Colorado Symptom Inventory. The Modified Colorado Symptom Inventory (MCSI; Conrad et al., 2001) is a brief clinician-administered measure of overall psychological distress. The 14-item measure has participants indicate the frequency with which they have experienced a number of interfering symptoms in the past month. Participants use a 5-point likert scale to respond to items $(0=$ not at all, 1 = once during the month, $2=$ several times during the month, $3=$ several times a week, $4=$ at least every day). A total score ranging from 0-56 is generated. The MCIS was adapted from the Colorado Symptom Inventory (CSI; Shern, Wilson, Coen, \& Patrick, 1994), a monitoring and outcome measure developed to describe the psychological distress experienced by a large community sample of individuals with SMI. The modified version differs from the original in that it has dropped non-scored follow-up questions about the level of interference of each queried symptom and one non-scored question regarding the impact of auditory hallucination. The dropped items either failed to demonstrate analytic integrity or were not administered consistently enough to provide evidence of their utility (Conrad et al., 2001). Additionally, the modified version altered the likert rating from 1-5 to 0-4, which altered total score ranges from 14-70 to $0-56$. The original CSI had an established 
clinical cut-off score of 30 (Boothroyd \& Chen, 2008). Though psychometric evaluations of the MCSI do not report a cut-off score, given the comparable scoring principles of the two measures, a clinical cut-off of 16 on the MCSI was used in the current study. The psychometric properties of the MCSI were investigated using 1,381 individuals from eight treatment sites. All participants were homeless or at risk for homelessness and had an SMI or substance abuse problem (or both). Results of this investigation showed that the MCSI is a valid and reliable measurement tool for psychological distress among SMI individuals. It demonstrated good convergent validity with another widely used measure of distress, the BSI $(r=.62)$. The internal consistency and test-retest rates were also favorable (intraclass correlation coefficient $=.79, r=.90$, respectively) (Conrad et al., 2001). The MCSI total score was used in the current study's analyses. It demonstrated an acceptable internal consistency rating $(\alpha=.87)$.

Simple Screening Instrument for Substance Abuse. The Simple Screening Instrument for Substance Abuse (SSI-SA; Winters \& Zenilman, 1994) is a 16-item self-report measure that assesses the potential for substance abuse and dependence. Participants complete 'yes' or 'no' questions about their use of substance and indicate experienced withdrawal symptoms by placing a 'check mark' beside the appropriate symptoms. Summing the endorsed items produces a total score that ranges from 0-14 ( 2 items are designated as filler and not scored). A cut-off score of 4 indicates possible problematic substance use. The SSI-SA is a widely used, governmentsupported questionnaire that demonstrates high sensitivity for the detection of substance use disorders (87-93\%) and excellent test-retest reliability $(r=.97)$ (Peters 
et al., 2000; Small, Simons, \& Stricherz, 2007). The SSI-SA total score was used in the current study's analyses. It demonstrated an acceptable internal consistency rating $(\alpha=.88)$

\section{Trauma variables.}

Lifetime Trauma Survey. The Lifetime Trauma Survey (LTS; Drescher, Hankin, \& Abueg, 1994) is a self-report measure that assesses the incidence of different traumatic events throughout one's lifetime. The domains of traumatic events that are queried include serious accident, disaster, childhood physical abuse, childhood sexual abuse, domestic violence, adult sexual assault, gang/drug-related violence, other criminal victimization, unexpected death of loved one, war trauma, racial/political/religious persecution, life threatening illness, and any other trauma not contained in the previous categories. Participants are asked to give a brief description of their experience and then indicate the frequency, chronicity, number of perpetrators, physical risk, and experienced emotional distress related to the traumas. This measure is used to collect trauma history information and does not compute subscales or scores.

\section{Data Analysis}

Following collection and cleaning of the data, potential confounding variables were examined. Independent T-tests and chi square analyses that compared the SMI and SMI-PTSD groups on demographic and diagnostic variables provided information on potential differences and subsequent confounds. These covariates were included in analyses when appropriate. In an effort to assure that all statistical 
procedures were appropriate for the type and scope of data collected, assumptions for the specific statistical tests were examined.

\section{Results}

\section{Descriptive}

Prior to formal hypothesis testing, statistical assumptions for normality, homegeneity, and multicollinearity were tested. Results of these analyses indicated that the data were in a tenable state for the planned tests of hypotheses. Specifically, normality distributions of the coping style subscales were examined with the the Shapiro-Wilk test, a powerful normality test that is most appropriately applied for small sample sizes (Razali \& Wah, 2011). Examinations of the overall data demonstrated that avoidance coping, as measured by the Brief COPE, $W(90)=.98, p$ $=.27$, and $\mathrm{CSC}, W(90)=.98, p=.20$ were normal. As were the problem-focused coping data, as measured by the Brief COPE, $W(90)=.99, p=.37$, and $\mathrm{CSC}, W(90)=$ $.95, p=.09$. Similarly, normality distributions of coping style subscales within study groups (SMI and SMI-PTSD) were normal. Taken together, no distribution inefficiencies were found within the study groups, nor the overall sample.

The total sample $(N=90)$ was represented with a fairly even split between males $(n=52,58 \%)$ and females $(n=38,42 \%)$. The average age of participants was 40 years old $($ range $=20-63$ years, $S D=10.7)$. Racial identity of participants included African American $(n=65,72 \%)$, Caucasian $(n=23,26 \%)$, and biracial $(n=2,2 \%)$. The most often reported educational achievement was graduation from high school or otherwise passing a General Educational Development (GED) test ( $n=28,35 \%)$. Most participants identified their relationship status as single $(n=74,82 \%)$ and 
reported that they were currently unemployed $(n=84,97 \%)$. The reported time since last employment varied from 1 month to 25 years, with an average of 54 months out of work $(S D=66.7)$. The majority acknowledged ever having legal problems $(n=68$, $83 \%)$, while a minority reported being currently on probation $(n=15,17 \%)$. Half of the total sample reported that they were staying at their own or someone else's apartment/home. The next most often reported housing statuses included sleeping outdoors $(n=22,24 \%)$ and short-term shelters $(n=13,14 \%)$. Slightly more than half of the sample ( $n=52,58 \%$ ) endorsed using alcohol or other substances within the past year. The overall sample's mean scores for substance abuse $(M=6.97, S D=$ 5.98), psychological distress (MCSI: $M=31.87, S D=11.52$ ), and PTSD symptom severity $(M=52.59, S D=5.62)$ were all above the clinical severity cutoffs. In terms of trauma exposure, the average number of unique traumas experienced by participants during their lifetime was $3.7(S D=2.20)$ and the most often reported traumas included domestic violence $(n=50,56 \%)$, serious accidents $(n=50,56 \%)$, criminal victimizations ( $n=41,46 \%)$, and childhood sexual abuse $(n=40,44 \%)$. Descriptives for the overall sample are represented in Table 3. 
Table 3

Demographic Characteristics of Participants $(N=90)$

\begin{tabular}{|c|c|c|}
\hline Characteristic & $n$ & $\%$ \\
\hline \multicolumn{3}{|l|}{ Sex } \\
\hline Male & 52 & 58 \\
\hline Female & 38 & 42 \\
\hline \multicolumn{3}{|l|}{ Race } \\
\hline African American & 65 & 72 \\
\hline Caucasian & 23 & 26 \\
\hline Bi-racial (AA and Asian) & 2 & 2 \\
\hline \multicolumn{3}{|l|}{ Housing } \\
\hline Outdoors & 22 & 24 \\
\hline Shelter (long or short-term) & 15 & 17 \\
\hline Own or other's home & 45 & 50 \\
\hline Hotel, boarding, halfway house & 8 & 9 \\
\hline \multicolumn{3}{|l|}{ Highest education level completed } \\
\hline $0-8$ th grade & 9 & 11 \\
\hline $9-11$ th grade & 25 & 31 \\
\hline High school or GED & 28 & 35 \\
\hline Some college & 10 & 12 \\
\hline Graduated college ( 2 or $4 y r)$ & 7 & 9 \\
\hline Graduate school & 2 & 3 \\
\hline \multicolumn{3}{|l|}{ Marital status } \\
\hline Single & 74 & 82 \\
\hline Married & 2 & 2 \\
\hline Divorced or separated & 11 & 12 \\
\hline Widowed & 3 & 3 \\
\hline Legal problems (ever) & 68 & 76 \\
\hline On probation at time of survey & 15 & 17 \\
\hline Alcohol or drug use in past year & 52 & 58 \\
\hline Trauma exposure & 75 & 83 \\
\hline Domestic violence & 50 & 56 \\
\hline Criminal victimization (e.g. mugging, etc.) & 41 & 46 \\
\hline Childhood sexual abuse & 40 & 44 \\
\hline Childhood physical abuse & 39 & 43 \\
\hline
\end{tabular}

Note. Totals of percentages are not 100 for every characteristic because of rounding. 
Demographic and descriptive characteristics between the study groups (SMI vs. SMI-PTSD) are displayed in Table 4. Significant differences were found between groups on sex, $\chi^{2}(1)=8.30, p<.05, \phi=.30$. Sex was further examined using an independent samples T-test to distinguish which cell contained disproportionate numbers and to examine sex as a potential confound for key variables. Results revealed no sex differences emerging across the two study groups and remaining key analysis variables. Subsequently, sex was not used in later analyses as a covariate. 
Table 4

Demographic Characteristics Between Groups

\begin{tabular}{|c|c|c|c|c|}
\hline \multirow[b]{2}{*}{ Characteristic } & \multicolumn{2}{|c|}{ SMI $(n=42)$} & \multicolumn{2}{|c|}{ SMI-PTSD $(n=48)$} \\
\hline & $n$ & $\%$ & $n$ & $\%$ \\
\hline \multicolumn{5}{|l|}{$\overline{S e x}$} \\
\hline Male & 31 & 74 & 21 & 44 \\
\hline Female & 11 & 26 & 27 & 56 \\
\hline \multicolumn{5}{|l|}{ Race } \\
\hline African American & 34 & 81 & 31 & 65 \\
\hline Caucasian & 8 & 19 & 15 & 31 \\
\hline Bi-racial (AA and Asian) & 0 & 0 & 2 & 4 \\
\hline \multicolumn{5}{|l|}{ Housing } \\
\hline Outdoors & 9 & 22 & 13 & 27 \\
\hline Shelter (long or short-term) & 6 & 14 & 9 & 19 \\
\hline Own or other's home & 22 & 52 & 23 & 48 \\
\hline Hotel, boarding, halfway house & 5 & 12 & 3 & 6 \\
\hline \multicolumn{5}{|l|}{ Highest education level completed } \\
\hline $0-8$ th grade & 3 & 8 & 6 & 14 \\
\hline $9-11$ th grade & 12 & 33 & 13 & 30 \\
\hline High school or GED & 13 & 35 & 15 & 34 \\
\hline Some college & 6 & 16 & 4 & 9 \\
\hline Graduated college ( 2 or $4 y r)$ & 2 & 5 & 5 & 11 \\
\hline Graduate school & 1 & 3 & 1 & 2 \\
\hline \multicolumn{5}{|l|}{ Marital status } \\
\hline Single & 37 & 88 & 37 & 77 \\
\hline Married & 0 & 0 & 2 & 4 \\
\hline Divorced or separated & 3 & 7 & 8 & 17 \\
\hline Widowed & 2 & 5 & 1 & 2 \\
\hline Legal problems (ever) & 32 & 82 & 36 & 84 \\
\hline On probation at time of survey & 6 & 15 & 9 & 19 \\
\hline Alcohol or drug use in past year & 22 & 52 & 30 & 63 \\
\hline Trauma exposure & 27 & 64 & 48 & 100 \\
\hline Domestic violence & 15 & 36 & 35 & 73 \\
\hline Criminal victimization (e.g. mugging, etc.) & 15 & 36 & 26 & 54 \\
\hline Childhood sexual abuse & 10 & 24 & 30 & 63 \\
\hline Childhood physical abuse & 11 & 26 & 28 & 58 \\
\hline
\end{tabular}

Note. Totals of percentages are not 100 for every characteristic because of rounding. 
Within the overall sample $(N=90)$, PTSD was the most often diagnosed condition $(n=48,53 \%)$. MDD was the most prevalent mood disorder $(n=33,37 \%)$. Schizophrenia was the most prevalent psychotic disorder within the sample $(n=24$, 27\%). Alcohol dependence was the most frequent substance use disorder, while cocaine dependence was the most frequent non-alcohol substance use disorder.

A summary of diagnostic frequencies between the two groups (SMI vs. SMIPTSD) is presented in Table 5 for descriptive purposes. Group comparisons by diagnosis were investigated to explore potential confounding diagnostic variables. Chi square results revealed significant differences between groups for $\operatorname{MDD} \chi^{2}(1)=$ $7.88, p<.05, \phi=.30$ with greater numbers of MDD diagnoses found in the SMIPTSD group $(n=24,50 \%)$. Schizophrenia diagnoses were also found to be significantly different between groups, $\chi^{2}(1)=26.63, p<.05, \phi=-.54$ with more schizophrenia diagnoses in the SMI group $(n=22,52 \%)$. Alcohol use was approaching significance $\chi^{2}(1)=3.72, p=.054, \phi=.20$, with more alcohol use disorders found in the SMI-PTSD group $(n=23,48 \%)$. T-tests were used to examine significant differences in coping, psychological distress, substance use, and posttraumatic symptoms based on the presence of MDD and schizophrenia. The results displayed in Tables 6 and 7 indicate that the means associated with key study variables, including avoidance coping, PTSD symptom severity, and psychological distress significantly differed depending on the presence or absence of these diagnoses. 
Table 5

Diagnostic Status at the time of Survey Between Groups

\begin{tabular}{|c|c|c|c|c|}
\hline \multirow[b]{2}{*}{ Diagnosis } & \multicolumn{2}{|c|}{$\operatorname{SMI}(n=42)$} & \multicolumn{2}{|c|}{ SMI-PTSD $(n=48)$} \\
\hline & $n$ & $\%$ & $n$ & $\%$ \\
\hline Major depressive disorder* & 9 & 21 & 24 & 50 \\
\hline Bipolar I disorder & 5 & 12 & 9 & 19 \\
\hline Dysthymic disorder & 1 & 2 & 2 & 4 \\
\hline Depressive disorder NOS & 1 & 2 & 0 & 0 \\
\hline Schizophrenia* & 22 & 52 & 2 & 4 \\
\hline Schizoaffective & 5 & 12 & 11 & 23 \\
\hline Delusional disorder & 1 & 2 & 1 & 2 \\
\hline Psychotic disorder NOS & 1 & 2 & 3 & 6 \\
\hline Schizoid personality disorder & 1 & 2 & 0 & 0 \\
\hline Schizotypal personality disorder & 1 & 2 & 0 & 0 \\
\hline Depression spectrum disorder* & 12 & 29 & 33 & 69 \\
\hline Psychotic spectrum disorder* & 30 & 71 & 15 & 31 \\
\hline Alcohol abuse or dependence* & 11 & 26 & 23 & 48 \\
\hline Drug abuse or dependence & 17 & 40 & 31 & 65 \\
\hline Panic disorder w/out agoraphobia* & 4 & 10 & 22 & 46 \\
\hline Social phobia & 7 & 17 & 12 & 25 \\
\hline Specific phobia & 1 & 2 & 3 & 6 \\
\hline Obsessive-compulsive disorder & 0 & 0 & 4 & 8 \\
\hline Generalized anxiety disorder & 0 & 0 & 2 & 4 \\
\hline Borderline personality disorder & 3 & 7 & 6 & 13 \\
\hline Antisocial personality disorder & 3 & 7 & 1 & 2 \\
\hline
\end{tabular}

*significant differences between groups (SMI vs. SMI-PTSD) observed at $p<.05$ 
Table 6

Major Depressive Disorder Diagnostic Presence by Key Variables

\begin{tabular}{|c|c|c|c|c|c|c|c|}
\hline \multirow[b]{2}{*}{ Key variables } & \multicolumn{2}{|c|}{$\begin{array}{l}\text { MDD present } \\
\quad(n=33)\end{array}$} & \multicolumn{2}{|c|}{$\begin{array}{l}\text { MDD absent } \\
\quad(n=57)\end{array}$} & \multirow[b]{2}{*}{$t(88)$} & \multirow[b]{2}{*}{$p$} & \multirow[b]{2}{*}{ Cohen's $d$} \\
\hline & $M$ & $S D$ & $M$ & $S D$ & & & \\
\hline \multicolumn{8}{|l|}{ Brief Cope } \\
\hline Problem-focused & 22.58 & 5.51 & 23.49 & 5.70 & 0.74 & 0.46 & -0.16 \\
\hline Avoidance & 27.45 & 5.47 & 22.65 & 6.10 & -3.73 & 0.00 & 0.83 \\
\hline \multicolumn{8}{|l|}{$\begin{array}{l}\text { Coping with Symptoms } \\
\text { Checklist }\end{array}$} \\
\hline Problem-focused & 1.44 & 0.46 & 1.76 & 0.57 & 2.70 & 0.01 & -0.61 \\
\hline Avoidance & 2.20 & 0.38 & 1.74 & 0.50 & -4.60 & 0.00 & 1.04 \\
\hline Posttraumatic Checklist & 62.45 & 15.33 & 46.88 & 18.64 & -4.07 & 0.00 & 0.91 \\
\hline $\begin{array}{l}\text { Modified Colorado } \\
\text { Symptoms Inventory }\end{array}$ & 37.94 & 6.66 & 28.35 & 12.29 & -4.14 & 0.00 & 0.97 \\
\hline $\begin{array}{l}\text { Brief Symptoms } \\
\text { Inventory }\end{array}$ & 2.58 & 0.83 & 1.76 & 1.08 & -3.76 & 0.00 & 0.85 \\
\hline $\begin{array}{l}\text { Simple Screening } \\
\text { Instrument }\end{array}$ & 8.49 & 6.11 & 6.09 & 5.78 & -1.86 & 0.07 & 0.40 \\
\hline
\end{tabular}

Note. MDD = Major depressive disorder 
Table 7

Schizophrenia Diagnostic Presence by Key Variables

\begin{tabular}{|c|c|c|c|c|c|c|c|}
\hline \multirow[b]{2}{*}{ Key variables } & \multicolumn{2}{|c|}{$\begin{array}{c}\text { Schizophrenia } \\
\text { present }(n=24)\end{array}$} & \multicolumn{2}{|c|}{$\begin{array}{c}\text { Schizophrenia } \\
\text { absent }(n=66)\end{array}$} & \multirow[b]{2}{*}{$t(88)$} & \multirow[b]{2}{*}{$p$} & \multirow[b]{2}{*}{ Cohen's $d$} \\
\hline & $M$ & $S D$ & $M$ & $S D$ & & & \\
\hline \multicolumn{8}{|l|}{ Brief Cope } \\
\hline Problem-focused & 24.25 & 5.83 & 22.76 & 5.54 & -1.12 & 0.27 & 0.26 \\
\hline Avoidance & 20.92 & 5.36 & 25.68 & 6.16 & 3.35 & 0.00 & -0.82 \\
\hline \multicolumn{8}{|l|}{$\begin{array}{l}\text { Coping with Symptoms } \\
\text { Checklist }\end{array}$} \\
\hline Problem-focused & 1.89 & 0.58 & 1.55 & 0.52 & -2.70 & 0.01 & 0.62 \\
\hline Avoidance & 1.49 & 0.47 & 2.06 & 0.43 & 5.40 & 0.07 & -1.27 \\
\hline Posttraumatic Checklist & 34.21 & 14.26 & 59.27 & 15.85 & 6.81 & 0.00 & -1.66 \\
\hline $\begin{array}{l}\text { Modified Colorado } \\
\text { Symptoms Inventory }\end{array}$ & 20.88 & 9.87 & 35.86 & 9.29 & 6.66 & 0.00 & -1.56 \\
\hline $\begin{array}{l}\text { Brief Symptoms } \\
\text { Inventory }\end{array}$ & 1.03 & 0.77 & 2.44 & 0.91 & 6.71 & 0.00 & -1.67 \\
\hline $\begin{array}{l}\text { Simple Screening } \\
\text { Instrument }\end{array}$ & 2.80 & 3.65 & 8.48 & 5.96 & 4.38 & 0.00 & -0.78 \\
\hline
\end{tabular}

In an effort to better understand the impact of these diagnoses on SMI and SMI-PTSD group differences a new variable was created that reports on whether participants had a primary depressive-spectrum disorder (i.e., MDD, bipolar I disorder, depressive disorder not otherwise specified, dysthymic disorder) or psychotic-spectrum disorder (i.e., schizophrenia, schizoaffective, delusional disorder, psychotic disorder not otherwise specified, schizoid personality disorder, schizotypal personality disorder). These transdiagnostic variables effectively capture the notable symptom differences between MDD and schizophrenia diagnoses. They also facilitate 
observation of the impact that diagnostic presence or absence has on all participants involved in the study, not just those with an MDD or schizophrenia diagnosis. These variables were included in relevant follow-up tests of hypotheses in order to account for any extraneous effects they may have had on the dependent variables being tested. Table 8 displays the categorical and dimensional composition of the spectrum disorders within each study group.

Table 8

Composition of Spectrum Disorders within Study Groups

\begin{tabular}{|c|c|c|c|c|c|c|}
\hline \multirow[b]{2}{*}{ Diagnostic Indicators } & \multicolumn{2}{|c|}{$\operatorname{SMI}(n=42)$} & \multicolumn{2}{|c|}{$\begin{array}{c}\text { SMI-PTSD }(n= \\
48) \\
\end{array}$} & \multicolumn{2}{|c|}{ Total $(N=90)$} \\
\hline & $n(\%)$ & $M(S D)$ & $n(\%)$ & $M(S D)$ & $n(\%)$ & $M(S D)$ \\
\hline Depressive-spectrum & $12(29 \%)$ & & $33(69 \%)$ & & $45(50 \%)$ & \\
\hline $\begin{array}{l}\text { Secondary psychotic- } \\
\text { spectrum disorder }\end{array}$ & $1(2 \%)$ & & $2(4 \%)$ & & $3(3 \%)$ & \\
\hline Trauma exposure & $9(21 \%)$ & & $33(69 \%)$ & & $42(47 \%)$ & \\
\hline Depression score & & $2.06(1.10)$ & & $3.06(0.91)$ & & $2.80(1.05)^{a}$ \\
\hline Psychoticism score & & $1.68(1.07)$ & & $2.58(0.95)$ & & $2.34(1.05)^{a}$ \\
\hline Psychotic-spectrum & $30(71 \%)$ & & $15(31 \%)$ & & $45(50 \%)$ & \\
\hline $\begin{array}{l}\text { Secondary depressive- } \\
\text { spectrum disorder }\end{array}$ & $3(7 \%)$ & & $0(0 \%)$ & & $3(3 \%)$ & \\
\hline Trauma exposure & $18(43 \%)$ & & $15(36 \%)$ & & $33(37 \%)$ & \\
\hline Depression score & & $1.28(1.16)$ & & $2.42(1.04)$ & & $1.66(1.23)^{b}$ \\
\hline Psychoticism score & & $1.33(1.04)$ & & $2.64(1.05)$ & & $1.77(1.20)^{b}$ \\
\hline
\end{tabular}

${ }^{a}$ represents mean score and standard deviation for depressive-spectrum group only ${ }^{b}$ represents mean score and standard deviation for psychotic-spectrum group only 
Aim 1. The first aim of the current investigation was to assess group differences in avoidance and problem-focused coping styles. The following key describes which measures and corresponding subscales were used in analyses that tested group differences in coping strategy use:

\begin{tabular}{|c|l|l|}
\hline \multicolumn{1}{|c|}{ Dependent variables } & \multicolumn{1}{|c|}{ Measure } & \multicolumn{1}{c|}{ Subscale } \\
\hline Avoidance coping & & \\
\hline Coping with SMI symptoms & CSC & Avoidance coping \\
\hline Coping with recent stressor & Brief-COPE & Avoidance coping \\
\hline Problem-focused coping & & \\
\hline Coping with SMI symptoms & CSC & Problem-focused coping \\
\hline Coping with recent stressor & Brief-COPE & Problem-focused coping \\
\hline
\end{tabular}

Hypothesis 1a. It was hypothesized that differences between SMI and SMIPTSD samples would emerge on measures of avoidance coping. Specifically, participants with an SMI-PTSD diagnosis would demonstrate increased avoidance coping as compared to those with SMI alone on measures of coping with SMI symptoms (CSC: avoidance subscale) and coping with recent stressors (Brief-COPE: avoidance subscale).

Hypothesis 1a was tested using a multivariate analysis of variance (MANOVA). MANOVA is a preferable test of group differences because it allows for simultaneous examination of several dependent variables between two groups without inflation of the family-wise error rate (Tabachnick \& Fidell, 2007). Two measures of avoidance coping were examined — one that assesses coping with SMI 
symptoms (CSC), and one that assesses coping with recent stressors (Brief-COPE).

The avoidance coping subscales represented the dependent variables in the analysis, with group (2 levels: SMI and SMI-PTSD) entered as the fixed factors. In the case of a significant omnibus effect, follow-up analysis of variance (ANOVA) tests with Bonferroni Corrections were conducted to determine the specific location of detected differences, and which dependent variables were implicated.

Result of the omnibus effect indicated that significant differences were found between the SMI and SMI-PTSD groups on measures of avoidance coping, Pillai's Trace $V=0.25, F(2,87)=14.79, p<.05$, partial $\eta^{2}=0.25$. Follow-up ANOVAs with Bonferroni Correction were performed to evidence specific group differences. Results indicated that avoidance coping, as measured by the Brief-COPE, was significantly greater among the SMI-PTSD group $(M=27.19, S D=4.86)$ as compared to the SMI group $(M=21.24, S D=6.30), F(1,88)=25.49, p<.05$, partial $\eta^{2}=0.23$. Additionally, avoidance coping, as measured by the CSC, was significantly greater for the SMI-PTSD group $(M=2.10, S D=0.38)$ as compared to the SMI group $(M=$ $1.68, S D=0.54), F(1,88)=18.68, p<.05$, partial $\eta^{2}=0.18$. Post hoc power analyses of this test indicated that with the total sample size $(N=90)$ and a moderate-large effect size $\left(f^{2}=0.34\right)$ this study had $99.9 \%$ power to detect group differences using two response variables at an alpha of .05. These MANOVA results support hypothesis 1a. Results are displayed in Table 9.

A follow-up MANOVA was conducted to observe main effect group differences (SMI vs. SMI-PTSD) on avoidance coping, while holding the presence of depressive-spectrum and psychotic-spectrum disorders constant. The transdiagnostic 
variable was entered as a fixed factor in the equation, along with the primary grouping variable, SMI vs. SMI-PTSD. The resulting MANOVA was a factorial $2 \times 2$ design. The ultimate goal of this test was to observe main effect group differences (SMI vs. SMI-PTSD) on avoidance coping, while holding the presence of depressivespectrum and psychotic-spectrum disorders constant.

Results of this follow-up test of omnibus effects upheld the original MANOVA findings, demonstrating that significant difference continued to be observed between the SMI and SMI-PTSD groups on measures of avoidance coping, though the effect was less robust, Pillai's Trace $V=0.16, F(2,85)=7.83, p<.05$, partial $\eta^{2}=0.16$. There were no significant interaction effects observed between study groups (SMI vs. SMI-PTSD) and spectrum disorders (depressive vs. psychotic spectrum). Follow-up ANOVAs with Bonferroni Correction were performed to evidence specific group differences. Results indicated that avoidance coping, as measured by the Brief-COPE, was significantly greater among the SMI-PTSD group $(M=27.19, S D=4.86)$ as compared to the SMI group $(M=21.24, S D=6.30), F(1$, $86)=14.45, p<.05$, partial $\eta^{2}=0.14$. Additionally, avoidance coping, as measured by the CSC, was significantly greater for the SMI-PTSD group $(M=2.10, S D=0.38)$ as compared to the SMI group $(M=1.68, S D=0.54), F(1,86)=7.75, p<.05$, partial $\eta^{2}=0.08$. These follow-up MANOVA results support hypothesis 1a.

Hypothesis 1 b. It was hypothesized that differences between SMI and SMIPTSD samples would emerge on measures of problem-focused coping. Specifically, participants with an SMI alone diagnosis would report increased use of problemfocused coping as compared to the SMI-PTSD group on measures of coping with 
SMI symptoms (CSC: problem-focused subscale) and coping with recent stressors (Brief-COPE: problem-focused subscale).

Hypothesis $1 \mathrm{~b}$ was tested using a MANOVA. Two measures of problemfocused coping were examined — one that assesses coping with SMI symptoms (CSC), and one that assesses coping with recent stressors (Brief-COPE). The problem-focused subscales represented the dependent variables in the analysis, with group (2 levels: SMI and SMI-PTSD) entered as the fixed factors. In the case of a significant omnibus effect between the groups, follow-up analysis of variance (ANOVA) tests with Bonferroni Corrections were conducted to determine the specific location of detected differences, and which dependent variables were implicated. Though a follow-up analysis was used to examine the impact of particular symptom structures (depression-spectrum and psychotic-spectrum) in hypothesis 1a, such tests were not necessary to examine the group differences posited in hypothesis 1b. This was because depressive and psychotic-spectrum diagnostic presence did not demonstrate similar confounding principles on problem-focused coping as they did on avoidance coping.

Results of this analysis did not indicate significant differences between the study groups, Pillai's Trace $V=0.06, F(2,87)=2.76, p=.07$, partial $\eta^{2}=0.06$. Follow-up ANOVAs with Bonferroni correction confirmed the non-significant differences between the groups on problem-focused coping as measured by the Brief$\operatorname{COPE}\left(F(1,88)=0.30, p=.60\right.$, partial $\left.\eta^{2}<0.00\right)$ and the $\operatorname{CSC}(F(1,88)=2.60, p=$ .11 , partial $\left.\eta^{2}=0.03\right)$. Post hoc power analyses of this test indicated that with the total sample size $(N=90)$ and a small effect size $\left(f^{2}=0.06\right)$ this study had $60 \%$ power 
to detect group differences using two response variables at an alpha of .05.

Hypothesis $1 \mathrm{~b}$ was not supported by the results. Results from hypotheses $1 \mathrm{a}$ and $1 \mathrm{~b}$ are displayed in Table 9.

Table 9

Means and Multivariate Analysis of Variance Results for Coping Strategies as a Function of Group $(N=90)$

\begin{tabular}{|c|c|c|c|c|c|c|c|}
\hline \multirow[b]{2}{*}{ Coping strategy } & \multicolumn{2}{|c|}{ SMI } & \multicolumn{2}{|c|}{ SMI-PTSD } & \multirow[b]{2}{*}{$F(1,88)$} & \multirow[b]{2}{*}{$p$} & \multirow[b]{2}{*}{ Partial $\eta^{2}$} \\
\hline & $M$ & $S D$ & $M$ & $S D$ & & & \\
\hline \multicolumn{8}{|l|}{ Brief-COPE } \\
\hline Avoidance & 21.24 & 6.30 & 27.19 & 4.86 & 25.49 & $<0.00$ & 0.23 \\
\hline Problem-focused & 22.80 & 5.80 & 23.46 & 5.50 & 0.30 & 0.59 & $<0.00$ \\
\hline \multicolumn{8}{|l|}{$\mathrm{CSC}$} \\
\hline Avoidance & 1.68 & 0.54 & 2.10 & 0.38 & 18.68 & $<0.00$ & 0.18 \\
\hline Problem-focused & 1.74 & 0.54 & 1.55 & 0.55 & 2.58 & 0.11 & 0.03 \\
\hline
\end{tabular}

Note. $\mathrm{CSC}=$ Coping with Symptoms Checklist

Aim 2. The second aim of the present study was to investigate differences in psychological distress as a function of PTSD diagnosis. Following the demonstration that those with SMI-PTSD report more psychological distress, the intention was to understand the role of coping in the relationship between PTSD symptom severity and psychological distress. The following key describes which measures and corresponding subscales were used as dependent variables and/or predictors in the analyses: 


\begin{tabular}{|l|l|l|}
\hline Dependent variable/predictor & \multicolumn{1}{|c|}{ Measure } & \multicolumn{1}{c|}{ Subscale } \\
\hline Psychological distress & BSI & Global severity index \\
\hline & MCSI & Total score \\
\hline PTSD symptom severity & PCL-S & Total score \\
\hline Avoidance coping & & \\
\hline Coping with recent stressor & Brief-COPE & Avoidance coping \\
\hline
\end{tabular}

Hypothesis 2a. It was hypothesized that on measures of overall psychological distress, individuals with SMI would report lower psychological distress as compared to their SMI-PTSD counterparts.

Hypothesis 2a was initially tested by conducting a MANOVA. Two measures of psychological distress were examined, both of which report on overall psychological distress--the BSI global severity index and the MCSI total score. These indices of psychological distress represented the dependent variables in the analysis, with group (2 levels: SMI and SMI-PTSD) entered as the fixed factors. Follow-up ANOVA tests with Bonferroni Corrections were conducted to determine the source of group differences on indices of psychological distress.

Results of this analysis indicated that significant differences were found between the SMI and SMI-PTSD groups on variables of psychological distress, Pillai's Trace $V=0.34, F(2,87)=22.61, p<.05$, partial $\eta^{2}=0.34$. Follow-up ANOVAs with Bonferroni Correction were performed to evidence specific group differences. Results indicated that overall psychological distress, as measured by the BSI, was significantly greater among the SMI-PTSD group $(M=2.64, S D=0.83)$ as compared to the SMI group $(M=1.40, S D=0.92), F(1,88)=45.68, p<.05$, partial $\eta^{2}=0.34$. Additionally, psychological distress as measured by the MCSI, was 
significantly greater for the SMI-PTSD group $(M=36.92, S D=8.83)$ as compared to the SMI group $(M=26.10, S D=11.59), F(1,88)=25.15, p<.05$, partial $\eta^{2}=0.22$. Post hoc power analyses of this test demonstrated that with the total sample size $(N=$ $90)$ and a large effect size $\left(f^{2}=0.34\right)$ this study had $99.9 \%$ power to detect group differences using two response variables at an alpha of .05. These MANOVA results support hypothesis 2a. Results are displayed in Table 10.

Table 10

Means and Multivariate Analysis of Covariance Results for Psychological Distress as a Function of Group

\begin{tabular}{lccccccccc}
\hline & \multicolumn{2}{c}{$\begin{array}{c}\text { SMI } \\
(n=42)\end{array}$} & & \multicolumn{2}{c}{$\begin{array}{c}\text { SMI-PTSD } \\
(n=48)\end{array}$} & & & \\
\cline { 2 - 3 } Psychological distress & $M$ & $S D$ & & $M$ & $S D$ & $F(1,85)$ & $p$ & Partial $\eta^{2}$ \\
\hline BSI & 1.40 & 0.92 & & 2.64 & 0.83 & 45.68 & $<0.00$ & 0.34 \\
MCSI & 26.10 & 11.59 & & 36.92 & 8.83 & 25.15 & $<0.00$ & 0.22 \\
\hline
\end{tabular}

Note. BSI $=$ Brief Symptom Inventory, MCSI = Modified Colorado Symptom Inventory

Similar to the follow-up analysis described in hypothesis 1a, a follow-up MANOVA was performed to test hypothesis $2 \mathrm{a}$. This was done to better understand the impact of depressive and psychotic symptom structures on SMI and SMI-PTSD group differences on psychological distress. The previously described transdiagnostic variable was entered as a fixed factor, along with the primary grouping variable, SMI vs. SMI-PTSD. Psychological distress measures were entered as dependent variables. The resulting MANOVA was a factorial 2x2 design. The ultimate goal of this test was to observe main effect group differences (SMI vs. SMI-PTSD) on psychological 
distress, while holding the presence of depressive-spectrum and psychotic-spectrum disorders constant. In the case of a significant omnibus effect between the groups, follow-up ANOVA tests with Bonferroni Corrections were conducted to determine the specific location of detected differences, and which measures of avoidance coping were implicated.

Results of this revised test of omnibus effect upheld the original MANOVA findings, demonstrating that significant difference continued to be observed between the SMI and SMI-PTSD groups on measures of psychological distress, though the effect was slightly less robust, Pillai's Trace $V=0.26, F(2,85)=15.16, p<.05$, partial $\eta^{2}=0.26$. There were no significant interaction effects observed between study groups (SMI vs. SMI-PTSD) and spectrum disorders (depressive vs. psychoticspectrum). Follow-up ANOVAs with Bonferroni Correction were performed to evidence specific group differences. Results indicated that psychological distress, as measured by the BSI, was significantly greater among the SMI-PTSD group $(M=$ 2.64, $S D=0.83)$ as compared to the SMI group $(M=1.40, S D=0.92), F(1,86)=$ $30.69, p<.05$, partial $\eta^{2}=0.26$. Additionally, psychological distress, as measured by the MCSI, was significantly greater for the SMI-PTSD group $(M=36.92, S D=8.83)$ as compared to the SMI group $(M=26.10, S D=11.59), F(1,86)=15.57, p<.05$, partial $\eta^{2}=0.15$. These follow-up MANOVA results support hypothesis $2 \mathrm{a}$.

Hypothesis 2 b: It was hypothesized that for individuals who have experienced a trauma, regardless of whether they met criteria for PTSD, PTSD symptom severity would predict overall psychological distress indirectly through avoidance coping. 
Previous hypotheses have relied on the designation of SMI versus SMI-PTSD grouping variables. For this particular hypothesis, SMI-PTSD participants were combined with SMI participants who endorsed 'Criteria A' trauma exposure $(n=75)$. This combined group was used to test the mediational impact of avoidance coping on the relationship between PTSD symptom severity and psychological distress. Baron and Kenny's (1986) statistical model of mediation was employed, with Preacher and Hayes's (2004) macro applied to test the model and its significance. Per Baron and Kenny's (1986) procedures for determining mediation, four steps were taken: (1) PTSD symptom severity (PCL-S total score), which is the independent variable, was regressed on psychological distress (BSI: global severity index), which is the dependent variable. (2) PTSD symptom severity was regressed on the mediator, avoidance coping (Brief-COPE: avoidance subscale). (3) Avoidance coping was regressed on psychological distress. (4) PTSD symptom severity was regressed on psychological distress, while controlling for the effect of avoidance coping. Full mediation is produced if the effect of PTSD symptom severity on psychological distress is reduced to zero upon the inclusion of avoidance coping. Partial mediation is produced if the effect of PTSD symptom severity on psychological distress, with avoidance coping involved, is reduced to a coefficient less than that produced in the first analysis (i.e. when avoidance coping is not involved in the equation). Finally, a Sobel Test was applied to find the significance of this indirect effect.

Results of the first analysis indicated that PTSD symptom severity significantly predicted psychological distress, $F(1,74)=125.09 p<.05, R^{2}=.63$. Results of the second analysis indicated that PTSD symptom severity significantly 
predicted avoidance coping, $F(1,74)=27.86 p<.05, R^{2}=.27$. Results of the third analysis indicated that avoidance coping significantly predicted psychological distress while controlling for PTSD symptom severity $F(1,74)=42.11 p<.05, R^{2}=.37$.

Results of the fourth regression were used to evaluate the overall mediational model. The findings demonstrated a reduction in the $\beta$ coefficient for this final model, but the prediction of PTSD symptom severity on psychological distress remained significant, $F(2,74)=76.46, p<.05, R^{2}=.68$. This reduced, but still significant $\beta$ coefficient indicated a partial mediation. Finally, a Sobel Test (Preacher \& Hayes, 2004) was applied to find the significance of this indirect effect. This test confirmed that the partial mediational effect was significant, $Z=2.76, p<.05$. Post hoc power analyses of this test indicated that with the included sample size $(n=75)$ and a large effect size $\left(R^{2}=.68\right)$ this study had $91.3 \%$ power to detect group differences using two predictor variables at an alpha of .05. Hypothesis $2 \mathrm{~b}$ was partially supported by the data. Results are displayed in Tables 11 and 12.

Table 11

Means, Standard Deviations, and Intercorrelations for Psychological Distress Predictor Variables Among Trauma Exposed Participants $(n=75)$

\begin{tabular}{lcccc}
\hline \multicolumn{1}{c}{ Variable } & $M$ & $S D$ & 1 & 2 \\
\hline Psychological distress & 2.24 & 1.00 & $0.79^{* * *}$ & $0.60^{* * *}$ \\
Predictor variable & & & & \\
1. PTSD symptom severity & 55.71 & 17.74 & -- & $0.53^{* * *}$ \\
2. Avoidance coping & & & & \\
\hline
\end{tabular}

${ }^{a}$ avoidance coping measured by Brief COPE subscale ${ }^{* * *} p<.001$ 
Table 12

Regression Analysis Summary for Predicting Psychological Distress Among Trauma Exposed Participants $(n=75)$

\begin{tabular}{|c|c|c|c|c|c|}
\hline Step and regression variables & $B$ & $S E B$ & $\beta$ & $t$ & $p$ \\
\hline \multicolumn{6}{|l|}{ Step 1: } \\
\hline $\begin{array}{l}\text { PTSD symptom severity on } \\
\text { psychological distress }\end{array}$ & 0.05 & 0.00 & 0.80 & 11.18 & $<0.00$ \\
\hline \multicolumn{6}{|l|}{ Step 2: } \\
\hline $\begin{array}{l}\text { PTSD symptom severity on } \\
\text { avoidance coping }\end{array}$ & 0.18 & 0.03 & 0.53 & 5.28 & $<0.00$ \\
\hline \multicolumn{6}{|l|}{ Step 3: } \\
\hline $\begin{array}{l}\text { Avoidance coping on psychological } \\
\text { distress, controlling for PTSD } \\
\text { symptom severity }\end{array}$ & 0.04 & 0.01 & 0.64 & 3.30 & 0.002 \\
\hline \multicolumn{6}{|l|}{ Step 4: } \\
\hline $\begin{array}{l}\text { PTSD symptom severity on } \\
\text { psychological distress, controlling } \\
\text { for avoidance coping }\end{array}$ & 0.04 & 0.00 & 0.26 & 8.40 & $<0.00$ \\
\hline
\end{tabular}

Note. $R^{2}=.68(N=75, p<.001)$.

Aim 3. The primary objective of the third aim was to investigate differences in substance abuse as a function of PTSD diagnostic status, and better understand the role of substance abuse in the relationship between PTSD symptom severity and avoidance coping separately for the groups. A secondary aim involved an examination of the relationship between criminal victimization and coping strategy use. The following key describes which measures and corresponding subscales were 
used as dependent variables and/or predictors in the analyses:

\begin{tabular}{|l|l|l|}
\hline \multicolumn{1}{|c|}{ Dependent variable/predictor } & \multicolumn{1}{|c|}{ Measure } & \multicolumn{1}{c|}{ Subscale } \\
\hline PTSD symptom severity & PCL-S & Total score \\
\hline Substance abuse & SSI-SA & Total score \\
\hline Avoidance coping & & \\
\hline Coping with recent stressor & Brief-COPE & Avoidance coping \\
\hline Criminal victimization & LTS & Trauma type \\
\hline & & Age of victimization \\
\hline & & Degree of distress \\
\hline
\end{tabular}

Hypothesis 3a: It was hypothesized that those with SMI-PTSD would show greater problematic use of substances compared to their SMI counterparts, such that a total substance abuse score would predict group membership.

To test this hypothesis, binary logistic regression was employed. Group membership (SMI vs. SMI-PTSD) represented the dependent variable, whereas total substance abuse score, as measured by the SSI-SA, was the predictor/covariate. The main effect of the predictor/covariate was evaluated by determining the significance of the resulting Wald statistic. Though performing a T-test would be an appropriate alternative to testing this hypothesis, binary logistic regression was chosen instead because the predictive ability of substance use was hypothesized to be robust enough to support a more rigorous test.

Initial examination of group mean differences revealed that the SMI-PTSD group $(M=9.08, S D=6.38)$ had significantly greater substance abuse scores as compared to the SMI group $(M=4.55, S D=4.44), t(84)=-3.95, p<.05, r=.40$. Results of the binary logistic regression showed that the substance abuse score was a 
significant predictor for group membership, Wald Statistic $=11.67, p<.05, R^{2}=.19$ and when added to the model increased group predictor classification from $53.3 \%$ to $62.2 \%$. The odds ratio $(O R)$ associated with the substance abuse coefficient $(O R=$ 1.16) indicated that for each point increase in the SSI-SA total score, an individual was 1.16 times as likely to belong to the SMI-PTSD group. This model explained between $14.3-19.1 \%$ of the variation in group membership. Post hoc power analyses of this test indicated that with the total sample size $(N=90)$ and a small effect size $(O R=1.16)$ this study had $26.1 \%$ power to predict group membership using one response variables at an alpha of .05. These findings support the hypothesis, though the effect size is small and group prediction was only slightly better than chance (62.2\%). Results are displayed in Table 13.

Table 13

Logistic Regression Predicting Group Membership without Covariates $(N=90)$

\begin{tabular}{lccccc}
\hline \multicolumn{1}{c}{ Predictor } & $B$ & $S E$ & $p$ & $O R$ & $95 \%$ CI \\
\hline Constant & -0.84 & 0.35 & 0.02 & & \\
Substance abuse score & 0.15 & 0.04 & $<0.00$ & 1.16 & {$[1.06,1.26]$} \\
\hline
\end{tabular}

Note. $\mathrm{CI}=$ confidence interval for odds ratio $(O R)$.

Similar to hypotheses 1a and 2a, this hypothesis included a follow-up test. This follow-up logistic regression was used to examine the impact of depressive and psychotic symptom structures on prediction of SMI and SMI-PTSD group membership. For this test, group membership (SMI vs. SMI-PTSD) represented the 
dependent variable. Substance abuse score was entered in the first block as the predictor/covariate. The second block included the previously described transdiagnostic variable entered as predictor/covariate. The transdiagnostic variable was entered into a block separate from the substance abuse predictor in order to evaluate both the effect of the transdiagnostic variables on the predictive model alone and when they are combined with substance abuse. The main effect of the predictors/covariates were evaluated by determining the significance of the resulting Wald statistic when all were entered into the model.

Results of this follow-up analysis demonstrate that upon the inclusion of the transdiagnostic variable into the model the -2 Log likelihood value dropped by 7.98 points, indicating a slightly better predictive model. The coefficient associated with substance abuse continued to be a significant predictor of group membership, Wald Statistic $=6.37, p<.05, O R=1.12$. This odds ratio indicated that for each increased point on the SSI-SA total score, an individual is 1.12 times as likely to belong to the SMI-PTSD group, while also considering the predictive impact of transdiagnostic spectrum disorder presence/absence in the model. Among the two transdiagnostic spectrums, only depressive-spectrum significantly contributed to the final model, Wald Statistic $=7.72, p<.05, O R=3.88$ and when added, increased group predictor classification from $62.2 \%$ to $71.1 \%$. This odds ratio indicated that individuals with a depressive-spectrum disorder present were nearly four times as likely to belong to the SMI-PTSD group, while also considering the predictive impact of substance abuse in the model. The final model, which includes substance use and transdiagnostic 
spectrum disorder presence/absence, explained between $21.6-28.8 \%$ of the variation in group membership.

Hypothesis 3b: It was hypothesized that PTSD symptom severity would predict avoidance coping indirectly through substance abuse for the SMI-PTSD group only.

This mediational model was tested on participants from the SMI-PTSD $(n=$ $48)$ and SMI $(n=42)$ groups separately. The hypothesized outcome was that only the SMI-PTSD group would evidence full mediation. For each group (SMI and SMIPTSD), mediation was tested according to Baron and Kenny's (1986) statistical model of mediation and the following steps were taken: (1) PTSD symptom severity (PCL-S total score), which is the independent variable, was regressed on avoidance coping (Brief COPE: avoidance subscale), which is the dependent variable. (2) PTSD symptom severity was regressed on the mediator, substance abuse (SSI-SA total score). (3) Substance abuse was regressed on avoidance coping. (4) PTSD symptom severity was regressed on avoidance coping, while controlling for the effect of substance abuse. Full mediation is produced if the effect of PTSD symptom severity on avoidance coping is reduced to zero upon the inclusion of substance abuse. Partial mediation is produced if the effect of PTSD symptom severity on avoidance coping, with substance abuse involved, is reduced to a coefficient less than that produced in the first analysis (i.e. when substance abuse is not involved in the equation). Finally, a Sobel Test was applied to find the significance of this indirect effect.

The first group of regression analyses were tested on the SMI group $(n=42)$. Results of the first analysis indicated that PTSD symptom severity $(M=39.21, S D=$ 
15.81) significantly predicted avoidance coping $(M=21.24, S D=6.30)$ for the SMI group, $F(1,41)=26.33 p<.05, R^{2}=.40$. Results of the second analysis indicated that PTSD symptom severity did not significantly predict substance abuse $(M=4.55, S D$ $=4.44)$ for the SMI group, $F(1,41)=1.61 p<.21, R^{2}=.04$. Given the lack of significant findings subsequent mediation model analyses were discontinued for the SMI group. Post hoc power analysis indicated that with the included sample size $(n=$ 42) and a small effect size $\left(R^{2}=.04\right)$ this study had $24.4 \%$ power to detect group differences using two predictor variables at an alpha of .05. Despite reduced power, these results partially support hypothesis $3 \mathrm{~b}$, which posited that only the SMI-PTSD group would evidence mediation.

The next set of regressions were conducted on the SMI-PTSD group $(n=48)$. Results of the first analysis indicated that PTSD symptom severity $(M=64.29, S D=$ 12.82) did not significantly predicted avoidance coping $(M=27.19, S D=4.86)$ for the SMI-PTSD group, $F(1,47)=0.20 p=.66, R^{2}=.00$. Given these non-significant findings, further mediation model analyses for the SMI-PTSD group were discontinued. Post hoc power analysis indicated that with the included sample size ( $n$ $=48)$ and a small effect size $\left(R^{2}=.00\right)$ this study had $16 \%$ power to detect group differences using two predictor variables at an alpha of .05. The reduced power, generated by low sample and effect sizes, were likely responsible for this nonsignificant finding. The data and results did not support hypothesis $3 \mathrm{~b}$.

Hypothesis 3c. Exploratory analysis of the relationship between victimization variables and coping strategies were conducted without specific directional hypotheses. Avoidance coping and problem-focused coping, both measured by the 
Brief COPE, were identified as the dependent variables in separate multiple regression analyses. Predictors entered into the model included trauma type (accident, natural disaster, child physical abuse, child sexual abuse, domestic violence, adult sexual assault, drug-related violence, and other criminal victimization), total unique traumas experienced, total unique traumas experienced as a child, adolescent, and adult, as well as PTSD symptom severity. Trauma type variables were represented with categorical data indicating the presence or absence (coded as 1 or 0 , respectively) of the traumatic event, while all other predictors were represented with continuous data. Two separate stepwise multiple regressions with all predictors entered collectively were conducted to test these variables' ability to predict both avoidance coping (Brief COPE: avoidance subscale) and problem-focused coping (Brief COPE: problem-focused subscale). Significant findings were explored with post-hoc tests.

Results of the multiple regression testing avoidance coping variance indicated that the linear combination of all 13 possible predictors was significantly related to avoidance coping $F(13,72)=4.103 p<.001, R^{2}=.43$. The model demonstrates that $43 \%$ of avoidance coping variance is attributable to the linear combination of 13 victimization variables. Further examination of the t-values and associated standardized beta weights of the predictors indicates a range of unique contributions from the predictors. PTSD symptom severity contributes the most to the prediction of variance in avoidance coping $(t(72)=5.13, p<.001, \beta=0.60)$. Total number of unique traumas is the other significant contributor to the model $(t(72)=-2.09, p<.05$, $\beta=-0.79)$. The signs associated with the t-values and beta weights of these predictors 
tell us that increased PTSD symptom severity is associated with predicting the variance of avoidance coping, whereas fewer total trauma types experienced predicts avoidance coping. Associated variable descriptors and results of the multiple regression for avoidance coping are displayed in Tables 14 and 15. 


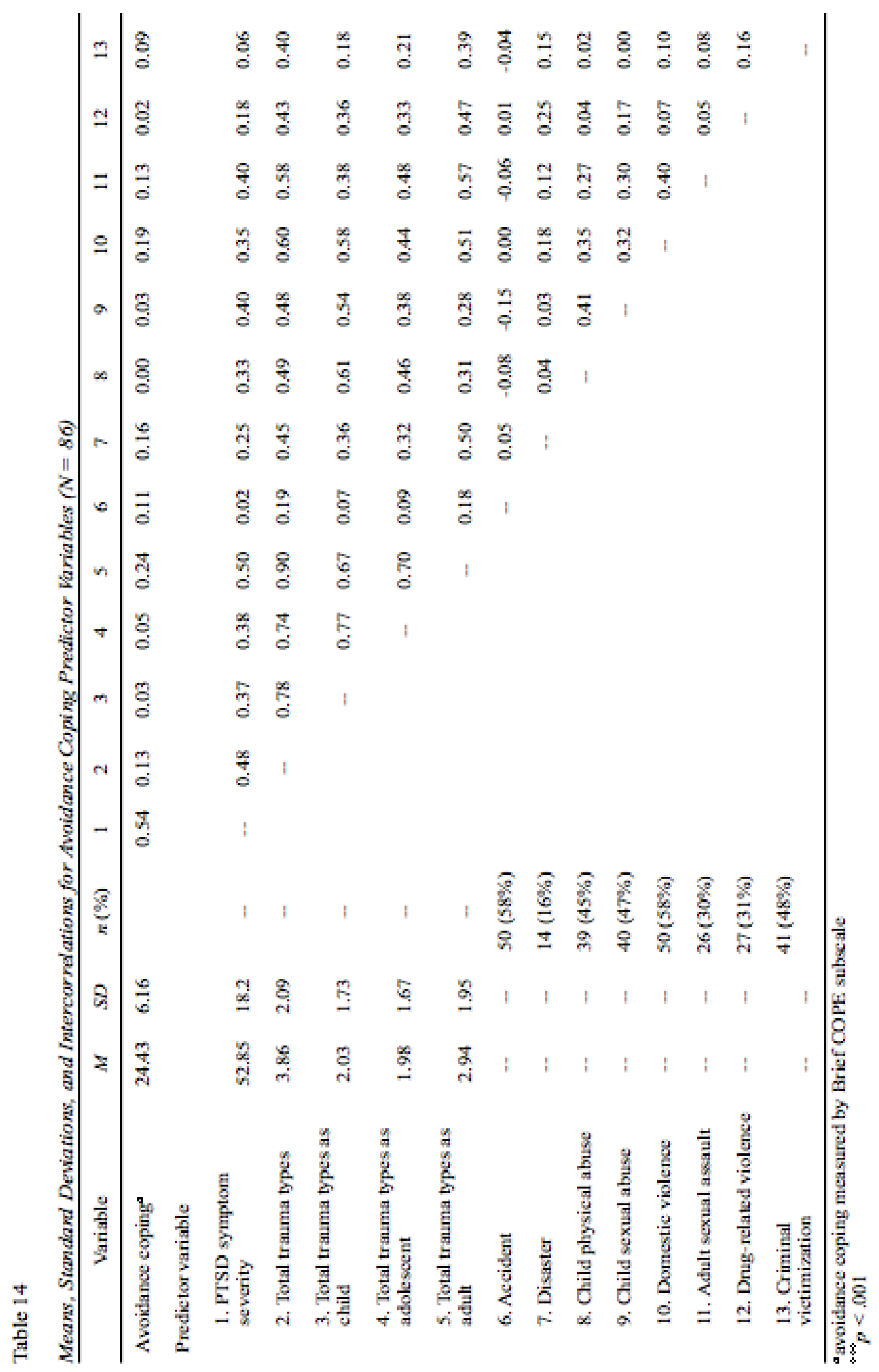


Table 15

Multiple Regression Analysis Summary for Predicting Avoidance Coping $(N=86)$

\begin{tabular}{lccccc}
\hline \multicolumn{1}{c}{ Regression variables } & $B$ & $S E B$ & $\beta$ & $t$ & $p$ \\
\hline Constant & 14.76 & 1.65 & -- & 8.97 & $<0.00$ \\
PTSD symptom severity & 0.21 & 0.03 & 0.63 & 6.48 & $<0.00$ \\
Total trauma types & -2.32 & 1.11 & -0.79 & -2.09 & $<0.00$ \\
Total trauma types as child & -0.33 & 0.72 & -0.09 & -0.45 & 0.04 \\
Total trauma types as adolescent & -0.39 & 0.58 & -0.11 & -0.68 & 0.50 \\
Total trauma types as adult & 1.26 & 0.84 & 0.40 & 1.49 & 0.14 \\
Accident & 2.63 & 1.48 & 0.21 & 1.78 & 0.08 \\
Disaster & 2.14 & 2.04 & 0.13 & 1.05 & 0.30 \\
Child physical abuse & 0.63 & 1.76 & 0.05 & 0.36 & 0.72 \\
Child sexual abuse & 0.64 & 1.73 & 0.05 & 0.37 & 0.71 \\
Domestic violence & 3.04 & 1.68 & 0.24 & 1.81 & 0.07 \\
Adult sexual assault & 0.73 & 1.88 & 0.05 & 0.39 & 0.70 \\
Drug-related violence & 0.45 & 1.65 & 0.03 & 0.27 & 0.79 \\
Criminal victimization & 2.59 & 1.52 & 0.21 & 1.71 & 0.09 \\
\hline & & & & & \\
\hline & & & & &
\end{tabular}

Results of the multiple regression testing problem-focused coping's variance indicated that the linear combination of all 13 possible predictors was significantly related to problem-focused coping $F(13,72)=2.05 p<.03, R^{2}=.27$. The model demonstrates that $27 \%$ of problem-focused coping variance is attributable to the linear combination of 13 victimization variables. Further examination of the t-values and associated standardized beta weights of the predictors indicates a range of unique contributions from the predictors. Exposure to general criminal victimization (e.g., 
robbery, mugging, etc.) contributes the most to the prediction of variance in problemfocused coping $(t(72)=-2.81, p<.01, \beta=-0.39)$. Total number of unique traumas as a child $(t(72)=2.23, p<.05, \beta=0.75)$, total number of number of unique traumas as an adult $(t(72)=2.11, p<.05, \beta=0.64)$, and exposure to natural disaster $(t(72)=-$ $2.05, p<.05, \beta=-0.28)$ are the other significant contributors to the model. The signs associated with the t-values and beta weights of these predictors tell us that exposure to trauma as a child and adult is associated with predicting the variance of problemfocused coping, while not being exposed to criminal victimization and natural disasters also predicts problem-focused coping. Associated variable descriptors and results of the multiple regression for problem-focused coping are displayed in Tables 16 and 17. 


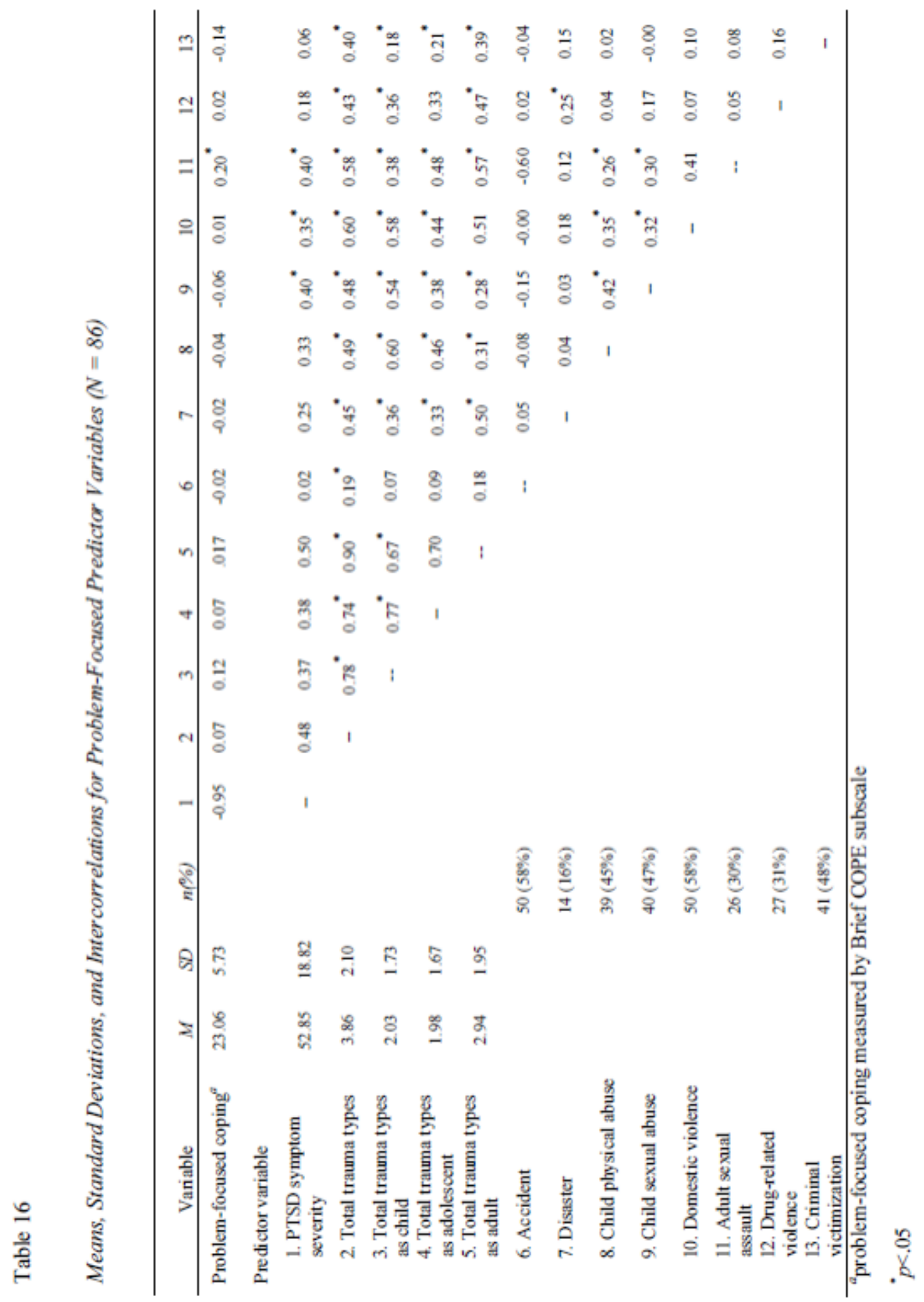


Table 17

Multiple Regression Analysis Summary for Predicting Problem-Focused Coping ( $N=$ 86)

\begin{tabular}{|c|c|c|c|c|c|}
\hline Regression variables & $B$ & $S E B$ & $\beta$ & $t$ & $p$ \\
\hline Constant & 26.66 & 2.03 & -- & 13.15 & $<0.00$ \\
\hline PTSD symptom severity & -0.06 & 0.04 & -0.20 & -1.51 & 0.14 \\
\hline Total trauma types & 0.52 & 1.16 & -0.19 & 0.45 & 0.66 \\
\hline Total trauma types as child & 1.68 & 0.75 & 0.51 & 2.23 & 0.03 \\
\hline Total trauma types as adolescent & -0.86 & 0.61 & -0.25 & -1.42 & 0.16 \\
\hline Total trauma types as adult & 1.87 & 0.88 & 0.64 & 2.12 & 0.04 \\
\hline Accident & -2.69 & 1.55 & -0.23 & -1.74 & 0.09 \\
\hline Disaster & -4.38 & 2.14 & -0.28 & -2.05 & 0.04 \\
\hline Child physical abuse & -2.98 & 1.84 & -0.26 & -1.62 & 0.11 \\
\hline Child sexual abuse & -2.67 & 1.81 & -0.23 & -1.47 & 0.14 \\
\hline Domestic violence & -3.30 & 1.76 & -0.29 & -1.88 & 0.06 \\
\hline Adult sexual assault & 0.62 & 1.97 & 0.05 & 0.32 & 0.75 \\
\hline Drug-related violence & -2.82 & 1.73 & -0.23 & -1.63 & 0.11 \\
\hline Criminal victimization & -4.46 & 1.59 & -0.39 & -2.81 & 0.01 \\
\hline
\end{tabular}

Post-hoc analyses. Given the previously described finding that certain depression and schizophrenia spectrum disorders were disproportionately dispersed between study groups and avoidance coping significantly differed depending on the disorders' presence or absence, further analyses were conducted in an effort to explore the predictive impact of study variables on coping strategy use. Recall that the current study's primary aims and hypotheses were tested using a transdiagnostic 
categorical variable to report on the impact of depression and schizophrenia-spectrum symptoms. This follow-up analysis used continuous measures of the spectrum symptoms by including the BSI subscales for depression and psychotic symptoms. The resulting analysis offers an alternative examination of the relationship between key study variables and coping strategy use. Two stepwise multiple regressions were conducted: one that predicted avoidance coping (Brief COPE: avoidance subscale) and one that predicted problem-focused coping (Brief COPE: problem-focused coping). The predictor variables used in each of the models included PTSD symptom severity (PCL-S), substance abuse (SSI-SA), overall psychological distress (MCSI: total score), depression symptoms (BSI: depression subscale), and psychotic symptoms (BSI: psychotic subscale). The dependent variables were avoidance coping and problem-focused coping. Only those with valid scores on all dependent variables were included in the analysis $(n=75)$.

Results of the first regression, which tested avoidance coping variance, indicated that of the possible predictors, one demonstrated significant predictive properties: depression symptoms. The resulting model demonstrated that depression symptoms accounted for $38.5 \%$ of the variability in avoidance coping, $F(1,73)=$ $45.65 p<.05, R^{2}=.39$. Examination of the $\mathrm{t}$-values and associated standardized beta weights further describes the impact of depression symptoms on prediction of variance in avoidance coping $(t(73)=6.76 p<.05, \beta=0.62)$. Results from this analysis are displayed in Tables 18 and 19. 
Table 18

Means, Standard Deviations, and Intercorrelations for Avoidance Coping Predictor

Variables $(n=75)$

\begin{tabular}{rccccccc}
\hline Variable & $M$ & $S D$ & 1 & 2 & 3 & 4 & 5 \\
\hline Avoidance coping $^{a}$ & 24.77 & 5.96 & $0.53^{* * *}$ & $0.29^{* *}$ & $0.44^{* * *}$ & $0.62^{* * *}$ & $0.55^{* * *}$
\end{tabular}

Predictor variable

1. PTSD symptom severity $55.7117 .74--\quad 0.29^{* *} 0.61^{* * *} 0.75^{* * *} 0.66^{* * *}$

2. Substance abuse

$7.71 \quad 6.05$

-- $0.41^{* * *} 0.41^{* * *} 0.33^{* *}$

3. Psychological distress ${ }^{b}$

$33.29 \quad 10.80$

$--0.73^{* * *} 0.73^{* * *}$

4. Depression symptoms

$2.37 \quad 1.23$

$-0.83^{* * *}$

5. Psychotic symptoms

$2.21 \quad 1.10$

avoidance coping measured by Brief COPE subscale

${ }_{*}^{b}$ psychological distress measured by MCSI total score

$\underset{* * *}{p}<.01$

$p<.001$

Table 19

Multiple Regression Analysis Summary for Predicting Avoidance Coping $(n=75)$

\begin{tabular}{lccccc}
\hline \multicolumn{1}{c}{ Regression variables } & $B$ & $S E B$ & $\beta$ & $t$ & $p$ \\
\hline Constant & 17.64 & 1.19 & -- & 14.85 & $<0.00$ \\
Depression symptoms & 3.01 & 0.45 & 0.62 & 6.76 & $<0.00$ \\
\hline
\end{tabular}




\section{Discussion}

The current study investigated coping strategy utilization by SMI individuals with and without comorbid PTSD, seeking services at a metro-area community mental health center. Overall, the sample $(N=90)$ reported high rates of trauma exposure, posttraumatic distress symptoms, psychological distress and substance abuse difficulties that fell within the ranges of clinical severity. Demographic descriptors of the sample indicated that a great proportion of participants have struggled with finances, employment, education, legal issues, and interpersonal relationships. These findings are in line with clinical and demographic findings from other investigations of SMI populations, which highlight the severity of the psychosocial struggles faced by those with SMI (Clark, Ricketts, \& McHugo, 1999; Ford, 2011; Hiday et al., 1999; Macdonald et al., 1998; Shelton et al., 2009).

The most frequent primary diagnoses carried by participants included MDD and schizophrenia. This is consistent with previous literature, which demonstrates that mood and psychotic disorders represent the most common diagnoses substantiating an SMI label (Drukker, van Os, Bak, Campo, \& Delespaul, 2010; Grubaugh, Cusack, \& Zinzow, 2008). However, the prevalence of schizophrenia in the overall sample $(n=$ $24,27 \%$ ) was lower than would be expected for a CMHC population. Findings from a 2010 census of Places for People demonstrate that $58 \%$ of their clientele hold a primary diagnosis of schizophrenia (Places for People, 2010). Disparate schizophrenia rates between the current study and the represented CMHC may limit the degree to which the current findings can be generalized to the larger CMHC population. A reduced rate of schizophrenia in the current study may have been a 
result of participant self-selection and/or interviewer selection based on severity of symptoms or distress. A subset of potential participants suffering from schizophrenia may have been particularly distressed or felt more distrustful of study involvement and chose not to participate. Alternatively, the study interviewer may have decided not to approach particularly severe or distressed clients with schizophrenia for participation, deciding that the individual would be better served by immediate clinical involvement rather than study participation at that time. Additionally, it is conceivable that a portion of individuals with schizophrenia who were connected to CMHC services were not willing or able to come to the center. While this portion would have been able to receive outreach services, not visiting the center's physical location barred them from potential participation in the study.

This study reveals the high rate of trauma exposure within a community-based sample of persons struggling with SMI. Overall, $83 \%$ of the total sample reported experiencing a 'Criteria $A$ ' traumatic event during their lifetime. The average number of different types of traumas experienced during one's lifetime was close to four, and the most often reported traumas included domestic violence $(n=50,56 \%)$, serious accidents $(n=50,56 \%)$, and childhood sexual abuse $(n=40,44 \%)$. The rate of trauma exposure within the current SMI sample rivals those estimates of the general population, which range from 39-56\% (Breslau et al., 1991; Kessler et al., 1995). They are consistent with previous investigations of SMI samples, the majority of which describe rates of lifetime trauma exposure greater than $85 \%$ (Cusack et al., 2006; Gearon et al., 2003; Lommen \& Restifo, 2009; Mueser et al., 1998; Subica et al., 2012). Trauma exposure had a significant impact on those included in this study, 
such that PTSD was the most prevalent psychiatric disorder diagnosed in the sample. Trauma exposure and PTSD rates described here support the accumulating evidence that SMI populations are much more likely to experience trauma and develop PTSD than the average person.

Also noteworthy, despite the significant psychiatric limitations of living with SMI, 36\% $(n=15)$ of those who have experienced a 'Criterion A' event did not go on to develop PTSD. Future research may seek to explore risk and resilience factors for the development of PTSD in this highly traumatized and psychiatrically compromised population.

The primary aims of this investigation were to examine coping strategy utilization as a function of PTSD diagnosis. Group differences between the SMI and SMI-PTSD samples emerged along a variety of examined dimensions. Most notable and consistent is the finding that avoidance coping is used by those with SMI-PTSD to a greater extent than individuals with SMI alone. This supports previous literature within the field of trauma study, which explains that avoidance is a prevalent emotional and behavioral strategy used by individuals with PTSD (Amir et al., 1997; Benotsch et al., 2000; Fortier et al., 2009; Galor \& Hentschel, 2012; Krause et al., 2008; Pineles et al., 2011; Schuettler \& Boals, 2011; Tiet et al., 2006). Though avoidance is known as a cluster of symptoms associated with PTSD, the current study reveals that it is also used by those with PTSD to negotiate non-trauma related life stressors and symptoms of their SMI.

A closer look at the SMI-PTSD sample's use of avoidance coping revealed a surprising finding. PTSD symptom severity did not significantly predict avoidance 
coping for those with SMI-PTSD. It was previously surmised that this group would score higher on avoidance coping due to the presence of their PTSD and associated symptoms. However, these findings suggest that it is not the severity of PTSD symptoms that is contributing to this group's increased use of avoidance coping. This indicates that for those with SMI-PTSD there are other, more powerful contributors that impact the extent to which they employ avoidance coping.

Depression symptoms may play an important role in avoidance coping. Posthoc regression analyses revealed that depression was the only significant predictor of avoidance coping among those who had experienced a 'criteria A' trauma. Taken together, these findings highlight the increased use of avoidance coping among those with a comorbid PTSD diagnosis, but also suggest that the depressive symptoms experienced by this group play a larger role in avoidance coping than PTSD symptoms alone.

Within the overall sample, avoidance coping mean scores were higher than problem-focused coping for management of SMI symptoms. These findings are inconsistent with other studies using the same measure. Yanos (2003) found within his community sample of SMI individuals $(N=91)$ that problem-focused coping was the more often used strategy. These inconsistent findings may be attributable to population differences between the studies. The most frequent SMI condition in Yanos's (2003) sample was schizophrenia, while the current study's was MDD. Further examination of the current sample revealed that among those with a schizophrenia/schizoaffective disorder, problem-focused coping usage was significantly higher than avoidance coping. This relative flip-flop in results for the 
overall versus schizophrenia/schizoaffective samples suggests that coping with symptoms and life stress may be more diagnosis-dependent than previously thought and highlights the limitations of using an umbrella-term, such as SMI to describe a varied population. Though this extends beyond the parameters of the current study's SMI vs SMI-PTSD scope, further investigation into coping style differences relative to specific SMI diagnoses could bring clarity to these issues.

Problem-focused coping did not emerge as a strategy that was used more often by a particular group. The mean scores between the two groups are fairly equal, indicating that problem-focused coping may be utilized to a similar extent by the groups. When comparing the problem-focused scores from the current study versus those published in the previous literature, few differences emerge. That is, our sample produced fairly similar mean scores for problem-focused coping as it relates to coping with SMI and coping with specific life stressors (Phillips et al., 2009; Pruessner et al., 2011; So \& Wong, 2008; Yanos et al., 2003). This indicates that our sample used problem-focused coping to a similar extent as some other SMI samples.

A number of victimization variables emerged as significant predictors of the variance attributable to problem-focused coping. Though the total variance explained by these variables was low, the combination of predictors was unexpected. Specifically, results indicated that the combination of having been exposed to increased child and adulthood traumas along with non-exposure to general criminal victimization and natural disasters came together to predict the use of problemfocused coping. This suggests that the timing of trauma exposure is particularly relevant for the use of problem-focused coping strategies. The interesting absence of 
adolescent trauma exposure as a significant predictor of problem-focused coping may indicate that those who use problem-focused coping did not experience chronic trauma exposure from childhood through adulthood. Alternatively, the data may simply demonstrate the relative non-importance of adolescent trauma exposure when it comes to the development and use of problem-focused coping. Further study of what is associated with and predicts problem-focused coping is needed to more fully understand this potentially beneficial coping style for those with SMI.

Posthoc analyses that examined the predictive ability of study variables on problem-focused coping were found to be particularly non-responsive. PTSD specific variables, along with substance use and indices of psychological distress failed to appropriately explain the variability associated with the use of problem-focused coping. Furthermore, limited sample sizes reduced the likelihood of generating significant findings, and as a result, problem-focused coping remains unrevealed in the context of this sample.

Psychological distress among participants was generally high and above clinical cut-offs for both groups. Hypothesis $2 \mathrm{a}$, which posits that there would be increased psychological distress among those with SMI-PTSD was upheld, indicating that the lasting effects of trauma exacerbate distress above and beyond that seen among individuals with SMI alone. Additionally, it was shown that PTSD symptom severity is a significant contributor to the psychological distress experienced by the trauma exposed participants. Although PTSD is not a disorder that has been traditionally considered an SMI, these findings indicate that the symptoms of PTSD have a significant impact on the overall distress experienced by those with SMIs. 
Further analyses demonstrated that the role of avoidance coping was partially responsible for how PTSD symptom severity influenced psychological distress. Taken together, for those who have been exposed to trauma, regardless of having a PTSD diagnosis, their psychological distress is at least partially accounted for by the ways they have been coping with difficult life experiences. This suggests that interventions aimed at mitigating distress following traumatic events may have a significant impact on the global distress experienced by those with SMI and possibly improve their overall level of functioning.

The findings from this investigation demonstrate that substance use and SUDs are problematic issues shared by individuals with SMI and PTSD, alike. In the current study, substance use is evaluated as it relates to PTSD diagnostic status and coping strategy utilization. Results indicated problematic substance use was significantly greater among the SMI-PTSD group and was distinctive enough to function as a predictor of group membership, albeit with limited effect size and power supporting the findings.

CMHCs are well positioned to recognize the increased rates of SUDs within their SMI clientele, and thereby offer assistance in the treating these disorders. Findings from the current investigation indicate that those reporting increased problematic substance use are at slightly increased risk to have an additional PTSD diagnosis on board. While the increased rates of PTSD among those with SMI are evidence enough to support the use of PTSD screening tools in CMHCs, these particular results support the use of such a measure as a follow-up to positive SUD 
screens. Use of such measures will aid in accurate and efficient identification and treatment of these taxing disorders.

Though relevant to the identification of a comorbid PTSD diagnosis, substance use did not emerge as an influential variable within the relationship between PTSD symptom severity and avoidance coping. These findings were surprising given the high comorbidities of SUDs and PTSD in previous studies (Christensen et al., 2005; Kessler et al., 1995; Mills, Teesson, Ross, \& Peters, 2006; O'Hare \& Sherrer, 2011; Ullman, Najdowski, \& Filipas, 2009; Yeater et al., 2010), as well as substance use being an identified avoidance coping strategy. However, at least one study, similar in design to the current investigation, reported a lack of significant associations between PTSD symptom severity and substance abuse among an SMI sample (Subica et al., 2012). The authors suggested that their brief SUD measure limited their findings. Measurement of problematic substance use in the current study did not seem to be a barrier to significant findings in these analyses as the measure is widely used and the overall sample endorsed problematic use within a range of clinical severity. Additionally, the data were normally distributed within groups. Given the low effect sizes attributed to these analyses, it is likely that the small samples sizes (SMI $n=42$; SMI-PTSD $n=48$ ) reduced statistical power and subsequently limited observable significant findings related to substance use.

\section{Implications and Future Directions}

The current investigation contributes to a line of burgeoning evidence that among those with SMI receiving services at CMHCs, rates of lifetime trauma exposure are significantly higher than the general population. These findings support 
recent discussion and efforts among community mental health researchers and clinicians to bring evidence-based trauma-focused services, including both assessment and treatment interventions to CMHCs (Christensen et al., 2005; Frueh, Grubaugh, Cusack, \& Elhai, 2009; Harris \& Fallot, 2001a, 2001b; Substance Abuse and Mental Health Services Administration, 2008, 2010). The recommendations supplied by these clinicians and organizations acknowledge the challenges associated with disseminating evidence-based practices within CMHCs and highlight the importance of multi-level staff trainings that work toward the development of a trauma-informed system of care.

Findings from the current investigation highlight the importance of coping, particularly avoidance coping, for those with SMI-PTSD diagnoses. This information is relevant to considerations for treatment. That is, psychotherapeutic interventions that have the potential to modify avoidance-based coping strategies may be particularly helpful for reducing psychological distress experienced by those with SMI-PTSD, though more research is needed to support this as a treatment recommendation for special populations.

Additionally, future investigations into psychological distress experienced by trauma exposed SMI populations may benefit from considering additional mediating factors alongside coping. In the current study, avoidance coping partially accounted for the relationship between PTSD symptom severity and psychological distress. Further investigation may uncover more and potentially stronger predictors that could influence future assessment and treatment recommendations for this deserving population. 
Finally, the use of SMI grouping terms has utility in defining a particularly burdened group, though it may limit the specificity of research findings. The current study noted disparities across the groups in mood and psychotic disorders. While efforts were taken within analyses to account for these differences, future studies may benefit from isolating particular diagnoses when considering conducting research on coping--a broad field of study in its own right.

\section{Limitations}

The current investigation had several limitations. First, the sample size was relatively small and limited the power of conducted analyses. This was perhaps most apparent in hypotheses that tested the attributable variance within problem-focused coping and the role of substance use in coping. A limited sample also impacts the degree to which findings can be generalized to the larger population of those with SMI and SMI-PTSD.

Diagnostic disparities between study groups proved to be a confounding factor that was reasonably addressed in analyses, but did not wholly answer questions about how findings may have been impacted by a more balanced diagnostic sample. Methodological solutions to arising diagnostic confounds may have been addressed by ongoing monitoring during the recruitment phase. This would have facilitated an earlier detection of diagnostic group differences and appropriate recruitment and/or assessment alterations could have been enacted. Specific recruitment changes may have included focusing study advertisements (i.e. flyers) on particular diagnoses that were under-represented or imbalanced between study groups, inviting CMHC clients who had previously established a connection with the center to participate in the 
study, or inviting back participants who were not previously able to sit for the study due to symptom distress or schedule conflicts. Specific alterations to assessment methodologies may have involved using the diagnostic tool to assess lifetime occurrence of Axis I and II disorders. Lifetime prevalence of SMI may have proved to be more balanced between study groups as compared to current diagnoses. Future studies involving recruitment of SMI individuals may find these strategies useful to balance study groups.

The demographic and socioeconomic assessment markers used in the current study were relatively broad. For example, determination of housing status included a combined category for living in one's own home and staying at another's home. These two groups may indicate drastic differences in housing, such that someone staying at another's home may be technically homeless, while someone with their own home is clearly not. Future studies may explore inclusion of more specific and reliable indicators of socioeconomic status. With more information about the social and contextual environments from which the sample is drawn we can better understand the influences of socioeconomic variables and lifestyle on variables of interest, including coping styles and their relative adaptiveness.

Another limitation of the study was that assessment administrators were not consistent throughout the course of the investigation. The final five participants recruited for the study were processed by a trained, but different research staff member. As previously described, analyses were conducted to examine potential differences between the participants assessed by the different staff and significant differences of moderate effect size were found. This inconsistency between staff 
made the results more susceptible to potential scientist-error, though no indication of this was made obvious in the data or results.

Scientific examination of coping strategies comes with a set of limitations. As previously described, there is a lack of methodological and measurement consistency within the field of coping literature (Phillips et al., 2009). Though the measures used in the current study were carefully chosen and empirically validated, the results of this study run the risk of contributing to a diffuse field of study and may not be easily integrated into future meta-analytic investigations of coping.

\section{Conclusion}

The current investigation demonstrates that the vast majority of individuals with SMI are likely to experience approximately four different types of traumatic events within their lifetime. This adds to accumulating evidence indicating that those with SMI and seeking services at CMHCs are more likely to have experienced trauma than the general population. Additionally, these findings support recommendations that CMHCs integrate evidence-based trauma-focused practices for appropriate assessment and treatment of their clientele.

Trauma exposed SMI individuals experience significant psychological distress that is strongly associated with the severity of their PTSD symptoms. This relationship between psychological distress and PTSD symptom severity is partially accounted for by the way they cope with stressful events, namely through avoidancebased coping. 
Avoidance coping is more prevalent among those with SMI-PTSD as compared to SMI alone. However, PTSD symptom severity does not have significant bearing on the extent to which those with SMI-PTSD use avoidance coping.

Psychological distress is higher among SMI-PTSD individuals than SMI alone. This supports the position that the additional experience of PTSD for those with an SMI leads to greater distress and potential symptom exacerbation.

The findings from this study add to the current literature on trauma exposure and coping as they relate to SMI populations. Attention to avoidance coping strategies within the context of treatment for SMI-PTSD may be a worthy treatment recommendation pending result replication and further investigation of the role of avoidance coping within an SMI population. 
References

Alvarez, M.-J., Roura, P., Foguet, Q., Oses, A., Sola, J., \& Arrufat, F.-X. (2012). Posttraumatic stress disorder comorbidity and clinical implications in patients with severe mental illness. Journal of Nervous and Mental Disease, 200(6), 549-552.

American Psychiatric Association. (2000). Diagnostic and Statistical Manual of Mental Disorders (4th, text revision ed.). In A. P. Association. (Ed.), Diagnostic and Statistical Manual of Mental Disorders (4th, text revision ed.). Washington, D.C.

Amir, M., Kaplan, Z., Efroni, R., Levine, Y., Benjamin, J., \& Kotler, M. (1997). Coping styles in post-traumatic stress disorder (PTSD) patients. Personality and Individual Differences, 23(3), 399-405. doi: 10.1016/s01918869(97)00066-4

Badour, C. L., Blonigen, D. M., Boden, M. T., Feldner, M. T., \& Bonn-Miller, M. O. (2012). A longitudinal test of the bi-directional relations between avoidance coping and PTSD severity during and after PTSD treatment. Behaviour Research and Therapy, 50(10), 610-616. doi: 10.1016/j.brat.2012.06.006

Baillargeon, J., Penn, J. V., Knight, K., Harzke, A. J., Baillargeon, G., \& Becker, E. A. (2010). Risk of reincarceration among prisoners with co-occurring severe mental illness and substance use disorders. Administration and Policy in Mental Health and Mental Health Services Research, 37(4), 367-374. doi: 10.1007/s10488-009-0252-9

Baron, R. M., \& Kenny, D. A. (1986). The moderator,Äìmediator variable distinction in social psychological research: Conceptual, strategic, and statistical considerations. Journal of Personality and Social Psychology, 51(6), 11731182. doi: 10.1037/0022-3514.51.6.1173

Benotsch, E. G., Brailey, K., Vasterling, J. J., Uddo, M., Constans, J. I., \& Sutker, P. B. (2000). War Zone stress, personal and environmental resources, and PTSD symptoms in Gulf War Veterans: A longitudinal perspective. Journal of Abnormal Psychology, 109(2), 205-213. doi: 10.1037/0021-843x.109.2.205

Berry, K., Barrowclough, C., Byrne, J., \& Purandare, N. (2006). Coping strategies and social support in old age psychosis. Social Psychiatry and Psychiatric Epidemiology, 41(4), 280-284. doi: 10.1007/s00127-005-0023-1

Betensky, J. D., Robinson, D. G., Gunduz-Bruce, H., Sevy, S., Lencz, T., Kane, J. M., .. . Szeszko, P. R. (2008). Patterns of stress in schizophrenia. Psychiatry Research, 160(1), 38-46.

Blanchard, E. B., Jones-Alexander, J., Buckley, T. C., \& Forneris, C. A. (1996). Psychometric properties of the PTSD Checklist (PCL). Behaviour Research and Therapy, 34(8), 669-673. doi: 10.1016/0005-7967(96)00033-2

Bokszczanin, A. (2003). The role of coping strategies and social support in adolescent's well-being after a flood. Polish Psychological Bulletin, 34(2), 6772 . 
Bonugli, R., Brackley, M. H., Williams, G. B., \& Lesser, J. (2010). Sexual abuse and posttraumatic stress disorder in adult women with severe mental illness: A pilot study. Issues in Mental Health Nursing, 31(7), 456-460. doi: $10.3109 / 01612840903581108$

Boothroyd, R. A., \& Chen, H. J. (2008). The psychometric properties of the Colorado Symptom Index. Administration and Policy in Mental Health and Mental Health Services Research, 35(5), 370-378. doi: 10.1007/s10488-008-0179-6

Brady, S., Rierdan, J., Penk, W., Losardo, M., \& Meschede, T. (2003). PostTraumatic Stress Disorder in Adults with Serious Mental Illness and Substance Abuse. Journal of Trauma \& Dissociation, 4(4), 77-90. doi: 10.1300/J229v04n04_06

Breslau, N., Davis, G. C., Andreski, P., \& Peterson, E. (1991). Traumatic events and posttraumatic stress disorder in an urban population of young adults. Archives of General Psychiatry, 48(3), 216-222.

Buckley, P. F., Miller, B. J., Lehrer, D. S., \& Castle, D. J. (2009). Psychiatric comorbidities and schizophrenia. Schizophrenia Bulletin, 35(2), 383-402. doi: $10.1093 / \mathrm{schbul} / \mathrm{sbn} 135$

Burton, E. G., Chaneb, B. J., \& Meeks, S. (2007). Factors related to coping with severe mental illness in the latter half of life. Clinical Gerontologist: The Journal of Aging and Mental Health, 30(4), 79-91. doi: 10.1300/J018v30n04 06

Capron, A. M. (1999). Ethical and human-rights issues in research on mental disorders that may affect decision-making capacity. The New England Journal of Medicine, 340(18), 1430-1434. doi: 10.1056/nejm199905063401812

Carver, C. S. (1997). You want to measure coping but your protocol's too long: Consider the Brief COPE. International Journal of Behavioral Medicine, 4(1), 92-100. doi: 10.1207/s15327558ijbm0401_6

Carver, C. S., Scheier, M. F., \& Weintraub, J. K. (1989). Assessing coping strategies: A theoretically based approach. Journal of Personality and Social Psychology, 56(2), 267-283. doi: 10.1037/0022-3514.56.2.267

Cascardi, M., Mueser, K. T., DeGiralomo, J., \& Murrin, M. (1996). Physical aggression against psychiatric inpatients by family members and partners. Psychiatric Services, 47(5).

Chilcoat, H. D., \& Menard, C. (2003). Epidemiological investigations: Comorbidity of posttraumatic stress disorder and substance use disorder. In P. Ouimette \& P. J. Brown (Eds.), Trauma and substance abuse: Causes, consequences, and treatment of comorbid disorders. (pp. 9-28). Washington, DC US: American Psychological Association.

Christensen, R. C., Hodgkins, C. C., Garces, L. K., Estlund, K. L., Miller, M. D., \& Touchton, R. (2005). Homeless, mentally ill and addicted: The need for abuse and trauma services. Journal of Health Care for the Poor and Underserved, 16(4), 615-621. doi: 10.1353/hpu.2005.0091

Ciapparelli, A., Paggini, R., Marazziti, D., Carmassi, C., Bianchi, M., Taponecco, C., ... Dell'Osso, L. (2007). Comorbidity with axis I anxiety disorders in remitted psychotic patients 1 year after hospitalization. CNS Spectrums, 12(12), 913919. 
Clark, R. E., Ricketts, S. K., \& McHugo, G. J. (1999). Legal system involvement an costs for persons in treatment for severe mental illness and substance use disorders. Psychiatric Services, 50(5), 641-647.

Classen, C. C., Palesh, O. G., \& Aggarwal, R. (2005). Sexual Revictimization: A Review of the Empirical Literature. Trauma, Violence, \& Abuse, 6(2), $103-$ 129. doi: $10.1177 / 1524838005275087$

Conrad, K. J., Yagelka, J. R., Matters, M. D., Rich, A. R., Williams, V., \& Buchanan, M. (2001). Reliability and validity of a modified Colorado Symptom Index in a national homeless sample. Mental Health Services Research, 3(3), 141-153. doi: 10.1023/a:1011571531303

Coverdale, J. H., \& Turbott, S. H. (2000). Sexual and physical abuse of chronically ill psychiatric outpatients compared with a matched sample of medical outpatients. Journal of Nervous and Mental Disease, 188(7), 440-445. doi: 10.1097/00005053-200007000-00008

Coyne, J. C., \& Racioppo, M. W. (2000). Never the twain shall meet? Closing the gap between coping research and clinical intervention research. American Psychologist, 55(6), 655-664. doi: 10.1037/0003-066x.55.6.655

Craine, L. S., Henson, C. E., Colliver, J. A., \& MacLean, D. G. (1988). Prevalence of a history of sexual abuse among female psychiatric patients in a state hospital system. Hospital \& Community Psychiatry, 39(3).

Cusack, K. J., Frueh, B., \& Brady, K. T. (2004). Trauma history screening in a community mental health center. Psychiatric Services, 55(2), 157-162.

Cusack, K. J., Grubaugh, A. L., Knapp, R. G., \& Frueh, B. C. (2006). Unrecognized trauma and PTSD among public mental health consumers with chronic and severe mental illness. Community Mental Health Journal, 42(5), 487-500. doi: 10.1007/s10597-006-9049-4

Derogatis, L. R. (1975). Brief Symptom Inventory. Baltimore, MD: Clinical Psychometric Research.

Derogatis, L. R. (1983). SCL-90-R, Administration, Scoring, and Procedures Manual (2nd Edition ed.). New York: Clinical Psychometric Research.

Derogatis, L. R., \& Melisaratos, N. (1983). The Brief Symptom Inventory: An introductory report. Psychological Medicine: A Journal of Research in Psychiatry and the Allied Sciences, 13(3), 595-605. doi: 10.1017/s0033291700048017

Drake, R. E., Essock, S. M., Shaner, A., Carey, K. B., Minkoff, K., Kola, L., . . Rickards, L. (2001). Implementing dual diagnosis services for clients with severe mental illness. Psychiatric Services, 52(4), 469-476. doi: 10.1176/appi.ps.52.4.469

Drake, R. E., \& Mueser, K. T. (2002). Co-Occurring Alcohol Use Disorder and Schizophrenia. Alcohol Research \& Health, 26(2), 99-102.

Drake, R. E., Mueser, K. T., Clark, R. E., \& Wallach, M. A. (2005). The Course, Treatment, and Outcome of Substance Disorder in Persons with Severe Mental Illness. In L. Davidson, C. Harding \& L. Spaniol (Eds.), Recovery from severe mental illnesses: Research evidence and implications for practice, Vol 1. (pp. 100-114). Boston, MA: Center for Psychiatric Rehabilitation/Boston U. 
Drukker, M., van Os, J., Bak, M., Campo, J., \& Delespaul, P. (2010). Systematic monitoring of needs for care and global outcomes in patients with severe mental illness. BMC Psychiatry, 10. doi: 10.1186/1471-244x-10-36

Fallot, R. D., \& Harris, M. (2001). A trauma-informed approach to screening and assessment.

Farhall, J., Greenwood, K. M., \& Jackson, H. J. (2007). Coping with hallucinated voices in schizophrenia: A review of self-initiated strategies and therapeutic interventions. Clinical Psychology Review, 27(4), 476-793. doi: 10.1016/j.cpr.2006.12.002

First, M. B., Gibbon M., Spitzer R. L., Williams, J. B., Benjamin L. S. (1997) Structured Clinical Interview for DSM-IV Axis II Personality Disorders, (SCID-II). Washington, D.C.: American Psychiatric Press, Inc.

First, M. B., Spitzer, R. L., Gibbon, M., \& Williams, J. B. W. (1996). User's guide for the Structured Clinical Interview for DSM-IV Axis I Disorders-Research Version (SCID-I). New York, NY: Biometrics Research Department.

Foa, E. B., \& Rothbaum, B. O. (1998). Treating the trauma of rape: Cognitivebehavioral therapy for PTSD. New York, NY US: Guilford Press.

Foa, E. B., Zinbarg, R., \& Rothbaum, B. O. (1992). Uncontrollability and unpredictability in post-traumatic stress disorder: An animal model. Psychological Bulletin, 112(2), 218-238. doi: 10.1037/0033-2909.112.2.218

Folstein, M. F., Folstein, S. E., \& McHugh, P. R. (1975). "Mini-mental state" : A practical method for grading the cognitive state of patients for the clinician. Journal of Psychiatric Research, 12(3), 189-198. doi: 10.1016/00223956(75)90026-6

Ford, J. D. (2011). Ethnoracial and educational differences in victimization history, trauma-related symptoms, and coping style. Psychological Trauma: Theory, Research, Practice, and Policy. doi: 10.1037/a0023670

Fortier, M. A., DiLillo, D., Messman-Moore, T. L., Peugh, J., DeNardi, K. A., \& Gaffey, K. J. (2009). Severity of child sexual abuse and revictimization: The mediating role of coping and trauma symptoms. Psychology of Women Quarterly, 33(3), 308-320. doi: 10.1111/j.1471-6402.2009.01503.x

Frueh, B., Grubaugh, A. L., Cusack, K. J., \& Elhai, J. D. (2009). Disseminating evidence-based practices for adults with PTSD and severe mental illness in public-sector mental health agencies. Behavior Modification, 33(1), 66-81. doi: $10.1177 / 0145445508322619$

Galor, S., \& Hentschel, U. (2012). Problem-solving tendencies, coping styles, and self-efficacy among Israeli veterans diagnosed with PTSD and depression. Journal of Loss and Trauma, 17(6), 522-535. doi: 10.1080/15325024.2012.674440

Ganguli, R., Singh, A., Brar, J., Carter, C., \& Mintun, M. (2002). Hydrocortisone induced regional cerebral activity changes in schizophrenia: A PET scan study. Schizophrenia Research, 56(3), 241-247. doi: 10.1016/s09209964(01)00219-5

Gearon, J. S., \& Bellack, A. S. (1999). Women with schizophrenia and co-occurring substance use disorders: An increased risk for violent victimization and HIV. 
Community Mental Health Journal, 35(5), 401-419. doi:

10.1023/a:1018778310859

Gearon, J. S., Kaltman, S. I., Brown, C., \& Bellack, A. S. (2003). Traumatic life events and PTSD among women with substance use disorders and schizophrenia. Psychiatric Services, 54(4), 523-528. doi:

10.1176/appi.ps.54.4.523

Glass, K., Flory, K., Hankin, B. L., Kloos, B., \& Turecki, G. (2009). Are coping strategies, social support, and hope associated with psychological distress among Hurricane Katrina survivors? Journal of Social and Clinical Psychology, 28(6), 779-795. doi: 10.1521/jscp.2009.28.6.779

Goodman, L. A., Rosenberg, S. D., Mueser, K. T., \& Drake, R. E. (1997). Physical and sexual assault history in women with serious mental illness: Prevalence, correlates, treatment, and future research directions. Schizophrenia Bulletin, 23(4), 685-696.

Goodman, L. A., Salyers, M. P., Mueser, K. T., Rosenberg, S. D., Swartz, M., Essock, S. M., . . . Swanson, J. (2001). Recent victimization in women and men with severe mental illness: Prevalence and correlates. Journal of Traumatic Stress, 14(4), 615-632. doi: 10.1023/a:1013026318450

Green, D. L., Choi, J. J., \& Kane, M. N. (2010). Coping strategies for victims of crime: Effects of the use of emotion-focused, problem-focused, and avoidance-oriented coping. Journal of Human Behavior in the Social Environment, 20(6), 732-743. doi: 10.1080/10911351003749128

Grubaugh, A. L., Cusack, K. J., \& Zinzow, H. M. (2008). "Trumping rules" affect diagnoses of persons with severe mental illness. Psychiatric Services, 59(10), 1219-1220. doi: http://dx.doi.org/10.1176/appi.ps.59.10.1219

Grubaugh, A. L., Zinzow, H. M., Paul, L., Egede, L. E., \& Frueh, B. C. (2011). Trauma exposure and posttraumatic stress disorder in adults with severe mental illness: A critical review. Clinical Psychology Review, 31(6), 883-899. doi: 10.1016/j.cpr.2011.04.003

Gutner, C. A., Rizvi, S. L., Monson, C. M., \& Resick, P. A. (2006). Changes in coping strategies, relationship to the perpetrator, and posttraumatic distress in female crime victims. Journal of Traumatic Stress, 19(6), 813-823. doi: $10.1002 /$ jts. 20158

Harris, M., \& Fallot, R. D. (2001a). Envisioning a trauma-informed service system: A vital paradigm shift. Harris, Maxine [Ed], 3-22.

Harris, M., \& Fallot, R. D. (2001b). Using trauma theory to design service systems. San Francisco, CA US: Jossey-Bass.

Hiday, V. A., Swartz, M. S., Swanson, J. W., Borum, R., \& Wagner, H. R. (1999). Criminal Victimization of Persons With Severe Mental Illness. Psychiatr Serv, 50(1), 62-68.

Hoe, M., \& Brekke, J. S. (2008). Cross-ethnic measurement invariance of the Brief Symptom Inventory for individuals with severe and persistent mental illness. Social Work Research, 32(2), 71-78.

Holahan, C. J., Moos, R. H., \& Schaefer, J. A. (1996). Coping, stress resistance and growth: Conceptualizing adaptive functioning. In M. Zeidner \& N. S. Endler 
(Eds.), Handbook of coping: Theory, research and applications (pp. 24-43). New York: John Wiley \& Sons.

Horan, W. P., Ventura, J., Mintz, J., Kopelowicz, A., Wirshing, D., ChristianHerman, J., . . L Liberman, R. P. (2007). Stress and coping responses to a natural disaster in people with schizophrenia. Psychiatry Research, 151(1-2), 77-86. doi: 10.1016/j.psychres.2006.10.009

Hultman, C. M., Wieselgren, I.-M., \& Ohman, A. (1997). Relationships between social support, social coping and life events in the relapse of schizophrenic patients. Scandinavian Journal of Psychology, 38(1), 3-13. doi: 10.1111/14679450.00002

Kessler, R. C., Sonnega, A., Bromet, E., Hughes, M., \& et al. (1995). Posttraumatic stress disorder in the National Comorbidity Survey. Archives of General Psychiatry, 52(12), 1048-1060.

Kessler, R. C., Wai, T. C., Demler, O., \& Walters, E. E. (2005). Prevalence, severity, and comorbidity of 12-month DSM-IV disorders in the National Comorbidity Survey Replication. Archives of General Psychiatry, 62(6), 617-627. doi: 10.1001/archpsyc.62.6.617

Kodesh, A., Goldshtein, I., Gelkopf, M., Goren, I., Chodick, G., \& Shalev, V. (2012). Epidemiology and comorbidity of severe mental illnesses in the community: Findings from a computerized mental health registry in a large Israeli health organization. Social Psychiatry and Psychiatric Epidemiology, 47(11), 17751782. doi: 10.1007/s00127-012-0478-9

Krageloh, C. (2011). A systematic review of studies using the Brief COPE: Religious coping in factor analyses. Religions, 2, 216-246.

Krause, E. D., Kaltman, S., Goodman, L. A., \& Dutton, M. A. (2008). Avoidant coping and PTSD symptoms related to domestic violence exposure: A longitudinal study. Journal of Traumatic Stress, 21(1), 83-90. doi: $10.1002 /$ jts. 20288

Kudoh, A., Ishihara, H., \& Matsuki, A. (1999). Pituitary-adrenal and parasympathetic function in chronic schizophrenic patients with postoperative ileus or hypotension. Neuropsychobiology, 39(3), 125-130. doi: 10.1159/000026572

Lazarus, R., \& Folkman, S. (1984). Stress, Appraisal, and Coping. New York: Springer Publishing.

Lobbestael, J., Leurgans, M., \& Arntz, A. (2011). Inter-rater reliability of the Structured Clinical Interview for DSM-IV Axis I Disorders (SCID-I) and Axis II Disorders (SCID-II). Clinical Psychology Psychotherapy, 18(1), 75-90. doi: 10.1002/cpp.693.

Lommen, M. J. J., \& Restifo, K. (2009). Trauma and posttraumatic stress disorder (PTSD) in patients with schizophrenia or schizoaffective disorder. Community Mental Health Journal, 45(6), 485-496. doi: 10.1007/s10597-009-9248-x

Lysaker, P. H., Wilt, M. A., Plascak-Hallberg, C. D., Brenner, C. A., \& Clements, C. A. (2003). Personality dimensions in schizophrenia: Associations with symptoms and coping. Journal of Nervous and Mental Disease, 191(2), 80-86. doi: 10.1097/00005053-200302000-00003

Macdonald, E. M., Jackson, H. J., Hayes, R. L., Baglioni, A. J., Jr., \& Madden, C. (1998). Social skill as a determinant of social networks and perceived social 
support in schizophrenia. Schizophrenia Research, 29(3), 275-286. doi: 10.1016/s0920-9964(97)00096-0

Maniglio, R. (2009). Severe mental illness and criminal victimization: A systematic review. Acta Psychiatrica Scandinavica, 119(3), 180-191. doi: 10.1111/j.1600-0447.2008.01300.x

McAlpine, D. D., \& Mechanic, D. (2000). Utilization of Specialty Mental Health Care Among Persons with Severe Mental Illness: The Roles of Demographics, Need, Insurance, and Risk. Health Services Research, 35(1), 277.

Meade, C. S., Kershaw, T. S., Hansen, N. B., \& Sikkema, K. J. (2009). Long-term correlates of childhood abuse among adults with severe mental illness: Adult victimization, substance abuse, and HIV sexual risk behavior. AIDS and Behavior, 13(2), 207-216. doi: 10.1007/s10461-007-9326-4

Meyer, B. (2001). Coping with severe mental illness: Relations of the Brief COPE with symptoms, functioning, and well-being. Journal of Psychopathology and Behavioral Assessment, 23(4), 265-277. doi: 10.1023/a:1012731520781

Mills, K. L., Teesson, M., Ross, J., \& Peters, L. (2006). Trauma, PTSD, and substance use disorders: Findings from the Australian National Survey of Mental Health and Well-Being. The American Journal of Psychiatry, 163(4), 651-658. doi: 10.1176/appi.ajp.163.4.652

Mueser, K. T., Goodman, L. B., Trumbetta, S. L., Rosenberg, S. D., Osher, C., Vidaver, R., .. . Foy, D. W. (1998). Trauma and posttraumatic stress disorder in severe mental illness. J Consult Clin Psychol, 66(3), 493-499.

Mueser, K. T., Rosenberg, S. D., Fox, L., Salyers, M. P., Ford, J. D., \& Carty, P. (2001). Psychometric evaluation of trauma and posttraumatic stress disorder assessments in persons with severe mental illness. Psychological Assessment, 13(1), 110-117. doi: 10.1037/1040-3590.13.1.110

Mueser, K. T., Rosenberg, S. D., Goodman, L. A., \& Trumbetta, S. L. (2002). Trauma, PTSD, and the course of severe mental illness: an interactive model. Schizophrenia Research, 53(1-2), 123-143. doi: 10.1016/s09209964(01)00173-6

Mueser, K. T., Rosenberg, S. D., Hamblen, J. L., \& Decamps, M. (2004). A cognitive-behavioral treatment program for posttraumatic stress disorder in persons with severe mental illness. American Journal of Psychiatric Rehabilitation, 7, 107-146. doi: 10.1080/1587760490476183

National Alliance on Mental Illness. (2011). What is Mental Illness: Mental Illness Facts. from http://www.nami.org/Content/NavigationMenu/Inform_Yourself/About_Ment al_Illness/About_Mental_Illness.htm

National Bioethics Advisory Commission. (1998). Research involving persons with mental disorders that may affect decisionmaking capacity. Rockville, MD: National Bioethics Advisory Commission.

National Institute of Mental Health. (2010). The Numbers Count: Mental Disorders in America. 2010, from http://www.nimh.nih.gov/health/publications/thenumbers-count-mental-disorders-in-america/index.shtml 
Nebioglu, M., \& Altindag, A. (2009). The prevalence of comorbid anxiety disorders in outpatients with schizophrenia. International Journal of Psychiatry in Clinical Practice, 13(4), 312-317. doi: 10.3109/13651500903094559

Nebraska Department of Health and Human Services. (2006). Nebraska Mental Health Board Training: Self Study Manual. Lincoln: Retrieved from http://www.dhhs.ne.gov/beh/Commit/Commit.htm.

Neria, Y., Bromet, E. J., Sievers, S., Lavelle, J., \& Fochtmann, L. J. (2002). Trauma exposure and posttraumatic stress disorder in psychosis: Findings from a firstadmission cohort. Journal of Consulting and Clinical Psychology, 70(1), 246251. doi: 10.1037/0022-006x.70.1.246

O'Hare, T., Shen, C., \& Sherrer, M. (2010). High-risk behaviors and drinking-to-cope as mediators of lifetime abuse and PTSD symptoms in clients with severe mental illness. Journal of Traumatic Stress, 23(2), 255-263.

O'Hare, T., \& Sherrer, M. (2011). Drinking motives as mediators between PTSD symptom severity and alcohol consumption in persons with severe mental illnesses. Addictive Behaviors, 36(5), 465-469. doi: 10.1016/j.addbeh.2011.01.006

O'Hare, T., Sherrer, M. V., \& Shen, C. (2006). Subjective Distress From Stressful Events and High-Risk Behaviors as Predictors of PTSD Symptom Severity in Clients With Severe Mental Illness. Journal of Traumatic Stress, 19(3), 375386. doi: $10.1002 /$ jts.20131

Ohio Department of Mental Health. (2004). Certification standards for community mental health agencies. Administrative rules 5122-23 to 5122-29. Columbus, $\mathrm{OH}$ : Author.

Peck, M. C., \& Scheffler, R. M. (2002). An analysis of the definitions of mental illness used in state parity laws. Psychiatric Services, 53(9), 1089-1095. doi: http://dx.doi.org/10.1176/appi.ps.53.9.1089

Penley, J. A., Tomaka, J., \& Wiebe, J. S. (2002). The association of coping to physical and psychological health outcomes: A meta-analytic review. Journal of Behavioral Medicine, 25(6), 551-603. doi: 10.1023/a:1020641400589

Peters, R. H., Greenbaum, P. E., Steinberg, M. L., Carter, C. R., Ortiz, M. M., Fry, B. C., \& Valle, S. K. (2000). Effectiveness of screening instruments in detecting substance use disorders among prisoners. Journal of Substance Abuse Treatment, 18(4), 349-358. doi: 10.1016/s0740-5472(99)00081-1

Phillips, L. J., Francey, S. M., Edwards, J., \& McMurray, N. (2009). Strategies used by psychotic individuals to cope with life stress and symptoms of illness: A systematic review. Anxiety, Stress \& Coping: An International Journal, 22(4), 371-410. doi: 10.1080/10615800902811065

Pineles, S. L., Mostoufi, S. M., Ready, C. B., Street, A. E., Griffin, M. G., \& Resick, P. A. (2011). Trauma reactivity, avoidant coping, and PTSD symptoms: A moderating relationship? Journal of Abnormal Psychology. doi: $10.1037 / \mathrm{a} 0022123$

Places for People. (2010). 2010 Fiscal Year Annual Report. St. Louis, MO: Places for People. 
Preacher, K. J., \& Hayes, A. F. (2004). SPSS and SAS procedures for estimating indirect effects in simple mediation models. Behavior Research Methods, Instruments \& Computers, 36(4), 717-731. doi: 10.3758/bf03206553

Pruessner, M., Iyer, S. N., Faridi, K., Joober, R., \& Malla, A. K. (2011). Stress and protective factors in individuals at ultra-high risk for psychosis, first episode psychosis and healthy controls. Schizophrenia Research, 129(1), 29-35. doi: 10.1016/j.schres.2011.03.022

Razali, N. M., \& Wah, Y. B. (2011). Power comparisons of Shapiro-Wilk, Kolmogorov-Smirnov, Lilliefors and Anderson-Darling tests. Journal of Statistical Modeling and Analytics, 2(1), 21-33.

Rea, L. M., \& Parker, R. A. (1992). Designing and conducting survey research. San Francisco: Jossey-Boss.

Resick, P. A., \& Schnicke, M. K. (1993). Cognitive processing therapy for rape victims: A treatment manual. Thousand Oaks, CA US: Sage Publications, Inc.

Rosenberg, S. D., Mueser, K. T., Friedman, M. J., Gorman, P. G., Drake, R. E., Vidaver, R. M., . . . Jankowski, M. K. (2001). Developing effective treatments for posttraumatic disorders among people with severe mental illness. Psychiatric Services, 52(11), 1453-1461. doi: http://dx.doi.org/10.1176/appi.ps.52.11.1453

Ruggiero, K. J., Del Ben, K., Scotti, J. R., \& Rabalais, A. E. (2003). Psychometric Properties of the PTSD Checklist--Civilian Version. Journal of Traumatic Stress, 16(5), 495-502. doi: 10.1023/a:1025714729117

Sabina, C., \& Tindale, R. S. (2008). Abuse characteristics and coping resources as predictors of problem-focused coping strategies among battered women. Violence Against Women, 14(4), 437-456. doi: 10.1177/1077801208314831

Schnider, K. R., Elhai, J. D., \& Gray, M. J. (2007). Coping style use predicts posttraumatic stress and complicated grief symptom severity among college students reporting a traumatic loss. Journal of Counseling Psychology, 54(3), 344-350. doi: 10.1037/0022-0167.54.3.344

Schuettler, D., \& Boals, A. (2011). The path to posttraumatic growth versus posttraumatic stress disorder: Contributions of event centrality and coping. Journal of Loss and Trauma, 16(2), 180-194. doi: $10.1080 / 15325024.2010 .519273$

Scott, S. T. (2007). Multiple traumatic experiences and the development of posttraumatic stress disorder. Journal of Interpersonal Violence, 22(7), 932938. doi: 10.1177/0886260507301226

Shelton, K. H., Taylor, P. J., Bonner, A., \& van den Bree, M. (2009). Risk factors for homelessness: Evidence from a population-based study. Psychiatric Services, 60(4), 465-472. doi: 10.1176/appi.ps.60.4.465

Shern, D. L., Wilson, N. Z., Coen, A. S., \& Patrick, D. C. (1994). Client outcomes: II. Longitudinal client data from the Colorado Treatment Outcome Study. Milbank Quarterly, 72(1), 123-148. doi: 10.2307/3350341

Small, N. J. K., Simons, J. S., \& Stricherz, M. (2007). Assessing criterion validity of the simple screening instrument for alcohol and other drug abuse (SSI-AOD) in a college population. Addictive Behaviors, 32(10), 2425-2431. doi: 10.1016/j.addbeh.2007.04.003 
So, S. H. W., \& Wong, C. W. (2008). Experience and coping with auditory hallucinations in first-episode psychosis: Relationship with stress coping. Hong Kong Journal of Psychiatry, 18(3), 115-121.

Subica, A. M., Claypoole, K. H., \& Wylie, A. M. (2012). PTSD'S mediation of the relationships between trauma, depression, substance abuse, mental health, and physical health in individuals with severe mental illness: Evaluating a comprehensive model. Schizophrenia Research, 136(1-3), 104-109. doi: 10.1016/j.schres.2011.10.018

Substance Abuse and Mental Health Services Administration. (2008). Jail Diversion and Trauma Recovery Program-Priority to Veterans

Short Title: Diversion and Trauma Recovery. 2011, from http://www.samhsa.gov/Grants/2008/sm_08_009.aspx

Substance Abuse and Mental Health Services Administration. (2010). The National Center for Trauma Informed Care. Retrieved November 3, 2010, from http://www.samhsa.gov/nctic/

Switzer, G. E., Dew, M. A., Thompson, K., Goycoolea, J. M., Derricott, T., \& Mullins, S. D. (1999). Posttraumatic stress disorder and service utilization among urban mental health center clients. Journal of Traumatic Stress, 12(1), 25-39. doi: http://dx.doi.org/10.1023/A:1024738114428

Swofford, C. D., Kasckow, J. W., Scheller-Gilkey, G., \& Inderbitzin, L. B. (1996). Substance use: A powerful predictor of relapse in schizophrenia.

Schizophrenia Research, 20(1-2), 145-151. doi: 10.1016/09209964(95)00068-2

Tarrier, N. (1987). An investigation of residual psychotic symptoms in discharged schizophrenic patients. British Journal of Clinical Psychology, 26(2), 141143.

Teplin, L. A., McClelland, G. M., Abram, K. M., \& Weiner, D. A. (2005). Crime Victimization in Adults With Severe Mental Illness: Comparison With the National Crime Victimization Survey. Archives of General Psychiatry, 62(8), 911-921. doi: 10.1001/archpsyc.62.8.911

Tiet, Q. Q., Rosen, C., Cavella, S., Moos, R. H., Finney, J. W., \& Yesavage, J. (2006). Coping, symptoms, and functioning outcomes of patients with posttraumatic stress disorder. Journal of Traumatic Stress, 19(6), 799-811. doi: 10.1002/jts.20185

Ullman, S. E., Filipas, H. H., Townsend, S. M., \& Starzynski, L. L. (2005). Trauma Exposure, Posttraumatic Stress Disorder and Problem Drinking in Sexual Assault Survivors. Journal of Studies on Alcohol, 66(5), 610-619.

Ullman, S. E., Najdowski, C. J., \& Filipas, H. H. (2009). Child sexual abuse, posttraumatic stress disorder, and substance use: Predictors of revictimization in adult sexual assault survivors. Journal of Child Sexual Abuse: Research, Treatment, \& Program Innovations for Victims, Survivors, \& Offenders, 18(4), 367-385. doi: 10.1080/10538710903035263

Virgo, N., Bennett, G., Higgins, D., Bennett, L., \& Thomas, P. (2001). The prevalence and characteristics of co-occurring serious mental illness (SMI) and substance abuse or dependence in the patients of adult mental health and 
addictions services in eastern Dorset. Journal of Mental Health, 10(2), 175188. doi: 10.1080/09638230125422

Vogel, M., Spitzer, C., Barnow, S., Freyberger, H. J., \& Grabe, H. J. r. (2006). The Role of Trauma and PTSD-Related Symptoms for Dissociation and Psychopathological Distress in Inpatients with Schizophrenia. Psychopathology, 39(5), 236-242. doi: 10.1159/000093924

Walser, R. D., \& Hayes, S. C. (2006). Acceptance and Commitment Therapy in the Treatment of Posttraumatic Stress Disorder: Theoretical and Applied Issues. In V. M. Follette \& J. I. Ruzek (Eds.), Cognitive-behavioral therapies for trauma (2nd ed.). (pp. 146-172). New York, NY US: Guilford Press.

Weathers, F. W., Litz, B. T., Herman, D. S., Huska, J. A., \& Keane, T. M. (1993). The PTSD Checklist (PCL): Reliability, validity, and diagnostic utility. Paper presented at the 9th annual meeting of the International Society for Traumatic Stress Studies, San Antonio, TX.

Welie, S. P. K., \& Berghmans, R. L. P. (2006). Inclusion of Patients with Severe Mental Illness in Clinical Trials: Issues and Recommendations Surrounding Informed Consent. CNS Drugs, 20(1), 67-83. doi: 10.2165/00023210200620010-00006

Whelan, P. J. P., Oleszek, J., Macdonald, A., \& Gaughran, F. (2009). The utility of the Mini-Mental State Examination in guiding assessment of capacity to consent to research. International Psychogeriatrics, 21(2), 338-344. doi: $10.1017 / \mathrm{s} 1041610208008314$

Winters, K., \& Zenilman, J. (1994). Simple screening instrument for outreach for alcohol and other drug abuse and infectious diseases. ((SMA) 02-3683). Rockville, MD: Center for Substance Abuse Treatment

Yanos, P. T., Knight, E. L., \& Bremer, L. (2003). A new measure of coping with symptoms for use with persons diagnosed with severe mental illness. Psychiatric Rehabilitation Journal, 27(2), 168-176. doi: 10.2975/27.2003.168.176

Yanos, P. T., West, M. L., \& Smith, S. M. (2010). Coping, productive time use, and negative mood among adults with severe mental illness: A daily diary study. Schizophrenia Research, 124(1-3), 54-59. doi: 10.1016/j.schres.2010.08.020

Yeater, E. A., Austin, J. L., Green, M. J., \& Smith, J. E. (2010). Coping mediates the relationship between posttraumatic stress disorder (PTSD) symptoms and alcohol use in homeless, ethnically diverse women: A preliminary study. Psychological Trauma: Theory, Research, Practice, and Policy, 2(4), 307310. doi: $10.1037 / \mathrm{a} 0021779$

Zanville, H. A., \& Cattaneo, L. B. (2009). Underdiagnosing and nontreatment of posttraumatic stress disorder in community mental health: A case study. Psychological Services, 6(1), 32-42. doi: http://dx.doi.org/10.1037/a0014410 